\title{
On the Feasibility of High Speed Railway mmWave Channels in Tunnel Scenario
}

\author{
Guangkai Li, ${ }^{1}$ Bo Ai, ${ }^{1,2}$ Danping He, ${ }^{1,2}$ Zhangdui Zhong, $^{1,2}$ Bing Hui, ${ }^{3}$ and Junhyeong Kim ${ }^{3,4}$ \\ ${ }^{1}$ State Key Laboratory of Rail Traffic Control and Safety, Beijing Jiaotong University, Beijing 100044, China \\ ${ }^{2}$ Beijing Engineering Research Center of High Speed Railway Broadband Mobile Communications, Beijing 100044, China \\ ${ }^{3}$ Mobile Application Research Department, Electronics and Telecommunications Research Institute (ETRI), \\ Daejeon 34129, Republic of Korea \\ ${ }^{4}$ School of Electrical Engineering, Korea Advanced Institute of Science and Technology (KAIST), Daejeon 34141, Republic of Korea
}

Correspondence should be addressed to Bo Ai; boai@bjtu.edu.cn

Received 21 May 2017; Revised 22 August 2017; Accepted 24 August 2017; Published 11 October 2017

Academic Editor: Francesco Benedetto

Copyright (C) 2017 Guangkai Li et al. This is an open access article distributed under the Creative Commons Attribution License, which permits unrestricted use, distribution, and reproduction in any medium, provided the original work is properly cited.

\begin{abstract}
Rail traffic is widely acknowledged as an efficient and green transportation pattern and its evolution attracts a lot of attention. However, the key point of the evolution is how to develop the railway services from traditional handling of the critical signaling applications only to high data rate applications, such as real-time videos for surveillance and entertainments. The promising method is trying to use millimeter wave which includes dozens of $\mathrm{GHz}$ bandwidths to bridge the high rate demand and frequency shortage. In this paper, the channel characteristics in an arched railway tunnel are investigated owing to their significance of designing reliable communication systems. Meantime, as millimeter wave suffers from higher propagation loss, directional antenna is widely accepted for designing the communication system. The specific changes that directional antenna brings to the radio channel are studied and compared to the performances of omnidirectional antenna. Note that the study is based on enhanced wide-band ray tracing tool where the electromagnetic and scattering parameters of the main materials of the tunnel are measured and fitted with predicting models.
\end{abstract}

\section{Introduction}

Mobile communication is one of the most essential and successful technology progress types, which becomes an indispensable part of more than 5 billion people [1]. With the mobile data demand exponentially growing [2-5], wireless communication systems working at millimeter wave (mmWave) have attracted much more attention in many mobile communication scenarios. For mmWave, it potentially contains a large amount of spectrum resources for achieving multi-Giga bps data rate for wireless communication systems [6-8].

As a typical application scene of mobile communication, high speed railway (HSR) with speed of more than $300 \mathrm{~km}$ per hour challenges the constantly improved mobile communication systems [9]. The most influential challenges for HSR mmWave communication system include frequent handovers, difficult signal processing as very high speed, and high penetration loss of signal from base-station to intrawagon user [10]. However, for achieving multi-Giga bps data rate in high speed railway (HSR), the existing HSR dedicated communication systems are no longer satisfactory. For example, the maximum data rate for the Global System for Mobile Radio Communications for Railway (GSM-R) is less than $200 \mathrm{kbps}$. Even for the Long Term Evolution for railway (LTE-R), it cannot provide more than $100 \mathrm{Mbps}$ data rate. Therefore, an interest group of High Rate Rail Communication (HRRC) has been established in the IEEE 802.15 working group for developing advanced mobile communication technologies, and it targets final achievement of very high data rate for HSR wireless communication system. Also, a distributed antenna system (DAS) [11] has been designed and it becomes a very promising communication system for HSR and metro system [10]. However, all these desiderate full understanding of the channel and an adequate and reliable channel model [12]. 
Since channel sounding measurements are treated as a priority when studying channels, in the era of $3 \mathrm{G}$ and $4 \mathrm{G}$ communication system, the channel parameters and models are extracted mostly from massive measurement campaigns [13]. But the measurement at mmWave band greatly challenges the existent sounding systems, especially for highdynamic channel measurement. The HSR mmWave channel measurement data is even rare [3]. So, we propose an approach that enhances the capability of deterministic channel model for obtaining mmWave HSR channel. Then, some important channel characteristics are studied and modeled for guiding future HSR mmWave communication system design.

In this paper, we simulate an important wireless channel by an enhanced ray tracing simulator where the electromagnetic (EM) and scattering parameters of dominant materials in the scenario are measured and fitted with suitable models. The simulation configuration practically follows the communication system demands in [15]. Meanwhile, a timeinterpolation method is employed for promoting the simulation capability for high-dynamic channels. Based on extensive simulations, the channel characteristics in an arched tunnel are completely exposed in two defined regions. Moreover, the channel feasibilities of using different antennas are investigated for determining the most suitable one.

The remainder of this paper is organized as follows: Section 2 describes principles of the proposed ray tracing tool with material measurement and time-interpolation. In Section 3, simulation scenario and system setups will be presented with more details. Section 4 demonstrates the feasibility of communication system with different antenna setups. Section 4 also illustrates the necessity of partition of regions in radio channel analysis. Then, characteristics of radio channel are provided in Section 5. Finally, Section 6 gives the conclusion.

\section{Ray-Optical Based mmWave Channel Modeling for High-Dynamic Scenario}

The wireless channel is simulated by utilizing ray-optical based deterministic propagation model, that is, ray tracing (RT) model. As it differs from other traditional RT, the enhanced RT used in this study has been calibrated in various frequencies and scenarios [16-19]. We make a special measurement for extracting electromagnetic (EM) and scattering parameters of the most influential materials in the HSR tunnel. The extracted parameters will be implemented into RT simulator. Moreover, for investigating the detailed channel characteristics in such high-dynamic scenario, a well-studied RT time-interpolation algorithm is employed to expose the channel small-scale characteristics.

The RT, in this study, is developed based on ray-optical algorithm [20] at Technische Universität Braunschweig [21]. It is three-dimension (3D) channel simulator which is performed in 3D digital map. For an integrated channel modeling, several radio propagation mechanisms are taken into consideration as line-of-sight (LOS) ray, reflection rays, and scattering rays. The LOS ray is known as free-space wave propagation between transmitter (TX) and receiver (RX). Its power is calculated by free-space path loss model in RT. The LOS path, if it exists, will dominate the received power in the $\mathrm{RX}$. Then, the reflection which is defined as the incident angle and reflected angle of EM wave from a surface is the same. Its power is calculated by solving the well-known Fresnel equations and the related reflection point in the $3 \mathrm{D}$ digital map is obtained by applying the image method [20]. Still, in the case of reflection, $n$th order multiple reflections will be practically considered for capturing dominant power contributions of total received power. For the diffuse scattering, the power contribution of diffuse scattering is evaluated by adopting the "effective roughness" (ER) scattering models [22]; the ER models generally include Lambertian Model, Directive Model, and Backscattering Lobe Model for predicting different types of scattering patterns [22]. Owing to the special structure of the tunnels where no obstacles are situated, the transmission through the materials and diffraction from edges of obstacles are omitted in the simulation.

Based on ray-optical principle and several propagation mechanism models, the final output of the RT is a timevariant channel impulse response (CIR) $h(\tau, t)$ which is the sum of powers of all the determined rays [23]:

$$
h(\tau, t)=\sum_{k=1}^{N(t)} a_{k}(t) \cdot e^{j\left(2 \pi f \tau_{k}(t)+\varphi_{k}(t)\right)} \cdot \delta\left(\tau-\tau_{k}(t)\right),
$$

where $a_{k}(t), \tau_{k}(t)$, and $\varphi_{k}(t)$ denote amplitude, delay, and additional phase shift of $k$ th ray (totally $N(t)$ rays that have been found by RT kernel).

Then $A_{k}(t)=a_{k}(t) \cdot e^{j\left(2 \pi f \tau_{k}(t)+\varphi_{k}(t)\right)}$ is defined as a complex coefficient; formula (1) can be rewritten as

$$
h(\tau, t)=\sum_{k=1}^{N(t)} A_{k}(t) \cdot \delta\left(\tau-\tau_{k}(t)\right)
$$

Then, (2) attached with the effects of wave polarization and antenna gains; $A_{k}(t)$ can be further expressed by

$$
\begin{aligned}
A_{k}(t)= & \sqrt{G_{\mathrm{RX}, k}} \cdot \vec{e}\left(\varphi_{\mathrm{RX}, k}, \theta_{\mathrm{RX}, k}\right)^{H} \cdot P_{k}(t) \cdot \sqrt{G_{\mathrm{TX}, k}} \\
& \cdot \vec{e}\left(\varphi_{\mathrm{TX}, k}, \theta_{\mathrm{TX}, k}\right) \cdot L_{k}(t),
\end{aligned}
$$

where $\varphi_{\mathrm{RX} / \mathrm{TX}, k}$ and $\theta_{\mathrm{RX} / \mathrm{TX}, k}$ indicate the angle of arrival (AOA) or angle of departure (AOD) of the $k$ th multipath component. Therefore, the vectors $\vec{e}\left(\varphi_{\mathrm{RX}, k}, \theta_{\mathrm{RX}, k}\right)$ and $\vec{e}\left(\varphi_{\mathrm{RX}, k}, \theta_{\mathrm{RX}, k}\right)$ denote the complex polarization vectors [24] of antennas at RX and TX, respectively. $G_{\mathrm{RX}, k}$ and $G_{\mathrm{TX}, k}$ indicate the additional antenna gains of the $k$ th multipath component in RX and TX. $(\cdot)^{H}$ denotes the Hermitian transpose. $P_{k}(t)$ depicts the complex channel polarization matrix which indicates the polarization shifting of the ray. $L_{k}(t)$ comprises propagation loss and the phase shift according to the delay $\tau_{k}(t)$ of the $k$ th multipath component. More information can be found in [21].

For investigating mmWave channel, it is still the challenge that the mmWave channel sounding measurements are very costly and time-consuming even for static scenarios. For measuring high-dynamic mmWave channel, it is even 


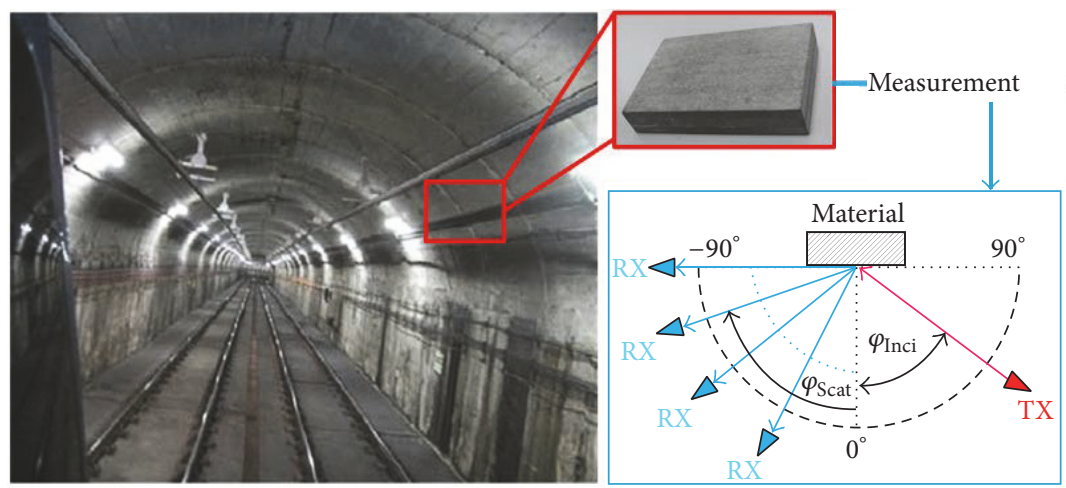

(a)

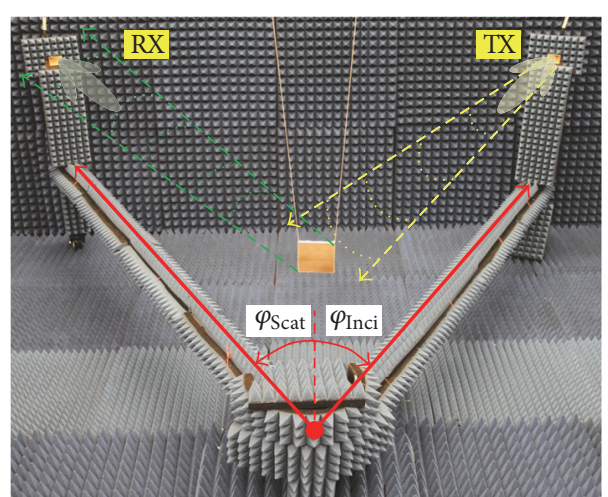

(b)

FIGURE 1: Study on EM and scattering parameters of cement wall. (a) Geometry of material study where the tunnel image is from [30]. (b) Measurement campaign.

unrealistic to measure a mmWave channel sample at a normal HSR speed (around $300 \mathrm{Km} / \mathrm{h}$ in China). So the RT is a promising tool for revealing the channel characteristics in HSR scenarios. It is also a trade-off between the limitation of measurement and demand for the channel data. To enhance the RT capability of accurately predicting channels in HSR tunnel scenario, we perform two approaches, as mentioned in the initial part of this chapter, which are measuring the EM and scattering parameters of the most influential material and utilizing RT time-interpolation algorithm to extract the small-scale channel parameters.

\subsection{EM and Scattering Parameters Acquisition of the Material.} The accurate energy calculations of the reflected and scattered rays in RT simulation depend on accurate EM (relative permittivity $\varepsilon_{r}$ in our study) and scattering parameters of materials as well as accurate 3D digital map of the scenario. Generally, RT simulation starts with determining the materials which constitute the scenario; then $\varepsilon_{r}$ and scattering parameters of these selected materials are acquired from checking literatures (e.g., ITU-R recommendations [25]), performing dedicated EM measurement [26], or derivation from channel measured data [27]. However, apart from dedicated EM measurement, these methods are less than ideal as $\varepsilon_{r}$ and scattering parameters of a material vary in different scenarios and conditions (e.g., humidity of environment and density of material). Therefore, we measure the interferences of the material on the wave propagation at $32.5 \mathrm{GHz}$ using a self-built testbed and estimate $\varepsilon_{r}$ and scattering parameters accordingly. And the material, in this study, is sulphoaluminate cement which is generally applied in HSR tunnels (cf. Figure 1(a)); unlike classic cerement utilized in buildings, it has advantages of quick-drying, high strength, compaction, and cementation which requires a dedicated measurement for studying its unknown $\varepsilon_{r}$ and scattering parameters.

As it is shown in Figure 1(b), the self-built testbed is on the basis of two high-accurate rotatable arms which could lead an accurate $2 \mathrm{D}$ scanning of the scattering of a material; the TX and RX are with horn antennas; and the Vector Network Analyzer (VNA) used in the measurement is manufactured by Keysight Corporation with model N5247A. The VNA measures $S$ - parameter between RF-ports of two cables after the end-to-end calibration process. In the measurement, the material is hanged upside the rotating center by a rope for eliminating some unwanted interference, which is consistent with the function of the anechoic chamber. As it is illustrated in the right of Figure 1(a), the diffuse scattering data is obtained by rotating the RX while the TX is fixed; and when $\varphi_{\text {Inci }}=\varphi_{\text {Scat }}$, we get the reflection data. After measurement, the data are postprocessed with proper filter to attenuate some interferences. Then, we can estimate $\varepsilon_{r}$ of the material by the method of free-space measurement based partly on [28]; and the estimated $\varepsilon_{r}$ is verified and slightly tuned with the measured refection coefficient accordingly (cf. Figure 2(a)). Meanwhile, the scattering parameters can be estimated by the method similar to $[22,26]$ but additionally with Simulated Annealing Algorithm [29] to obtain the better scattering parameters by automatic minimising of the gap between fitting results and measurement (cf. Figure 2(b)). It should be noted that the Directive Model as one of most important ER models was employed to fit the measured scattering radiation patterns; therefore frequency-dependent $S(f)$ and $\alpha_{R}(f)$ of Directive Model are the scattering parameters that should be extracted from estimation process $[22,26]$.

As it is described above, a brick made of sulphoaluminate cement was measured; Figure 2 illustrates the estimation processes of relative permittivity $\left(\varepsilon_{r}\right)$ and scattering parameters $\left(S\right.$ and $\left.\alpha_{R}\right)$ at $32.5 \mathrm{GHz}$. Figure 2(a) gives the comparison of reflection coefficients between fitting curve and measurement. $\varepsilon_{r}$ of the fitting curve is $\varepsilon_{r}=3.47-j 0.15$, where the mean error (ME) and standard deviation (Std) between measurement and fitting curve are 0.0070 and 0.0141 , respectively. Furthermore, the Directive Model is fitted with measurement in various incident positions; for example, Figure 2(b) gives a scattering fitting curve when $\varphi_{\text {Inci }}=70^{\circ}$. The most suitable $S$ and $\alpha_{R}$ for the scattering fitting are 0.00118 and 120 , respectively. The ME and Std between fitting curve and measurement are $2.16 e-4$ and $5.77 e-4$, respectively.

2.2. Interpolation Algorithm in RT. The main drawback of the RT is the high computational cost according to the complexity of $3 \mathrm{D}$ digital map. For dynamic channel 


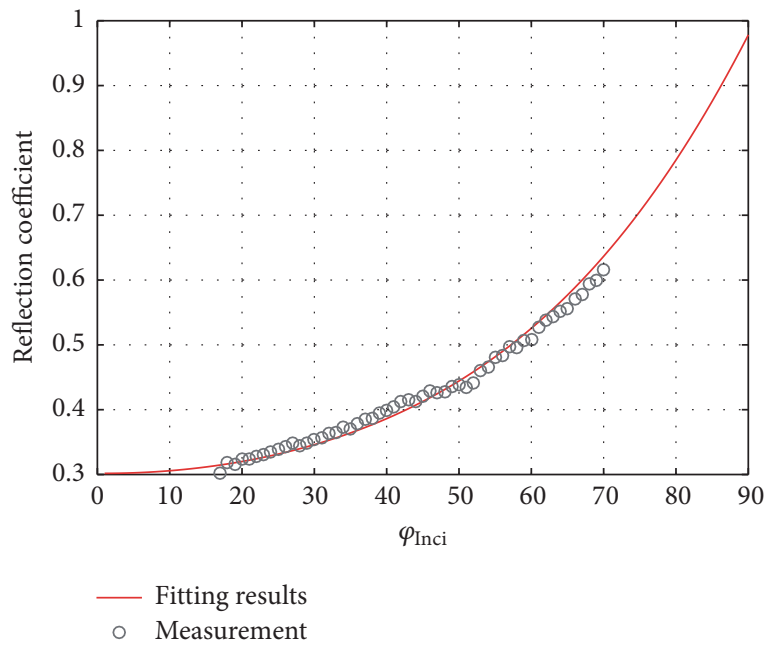

(a)

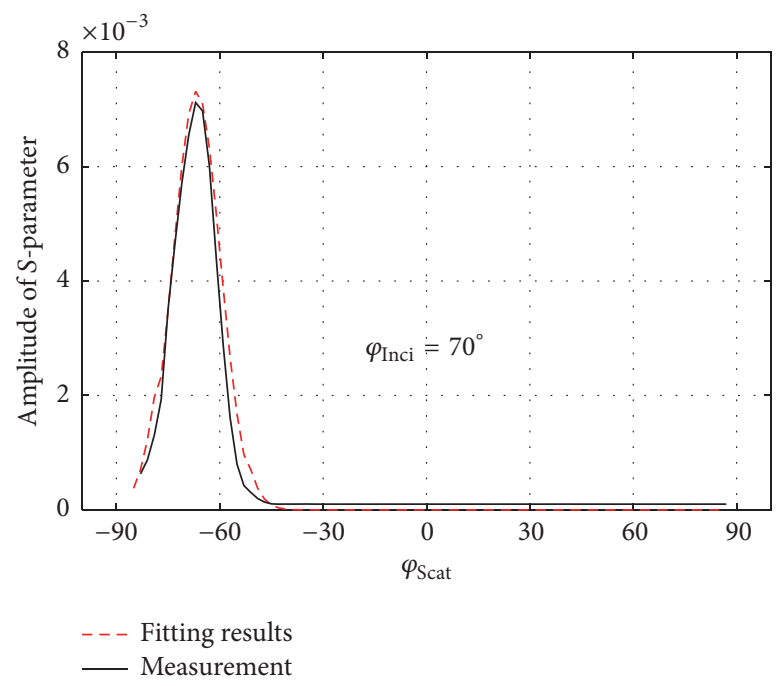

(b)

FIGURE 2: The comparison between fitting curve and measurement of cement brick at $32.5 \mathrm{GHz}$. (a) Reflection. (b) Scattering.

characteristics learning, especially the HSR channel, a high time resolution $\left(\Delta t=t_{n}-t_{n-1}\right)$ of the channel is required for the study of both large-scale and small-scale channel characteristics. In this study, the distance between TX and $\mathrm{RX}$ ranges from zero to one kilometer $(\mathrm{km})$. As the channel at $30 \mathrm{GHz}$ band is studied, the distance interval between $h\left(\tau, t_{n}\right)$ and $h\left(\tau, t_{n-1}\right)$ should be small enough (normally less than half-wavelength of EM wave which is 5 millimeter $(\mathrm{mm})$ in this study [31]). The geometry-based path interpolation is employed to overcome the impractical simulation time due to the high computational complexity [32]. The basic idea of the interpolation is to obtain the information about the continuous propagation paths between two consecutive scenario snapshots. Then the linear interpolation will be performed between the two continuous paths. The detailed description of this algorithm is in [32].

For this simulation in an arched tunnel, the initial time resolution is 10 milliseconds $(\mathrm{ms})$ in the simulation, so the initial interval between two sampled snapshots is $1 \mathrm{~m}$ as the speed of HSR is assumed to be $360 \mathrm{~km} / \mathrm{h}$ in this study. Then, the interpolation algorithm is preformed between each two snapshots. By utilizing geometry-based ray information, the method of interpolation will drop time resolution to $2 \mathrm{~ms}$ ( $2 \mathrm{~mm}$ interval in distance) in this study. Therefore, with very small time resolution, the extraction of small-scale fading parameters can be guaranteed.

\section{Simulation Scenario and System Setups in the Tunnel}

3.1. Tunnel Scenario in Simulation. In this study, the straight arched tunnel is employed as the HSR tunnel scenario. Figure 3 shows the overview of the tunnel scenario in the simulation. Figure 3(a) illustrated that the cross section of tunnel includes the accurate dimension and the locations of TX1 and RX1. According to realistic "Type II" tunnel described in [14], the arched tunnel in this study is with dimension $8.41 \mathrm{~m} \times 6.87 \mathrm{~m}\left(W_{\text {tunnel }} \times H_{\text {tunnel }}\right.$, where $W_{\text {tunnel }}$ and $H_{\text {tunnel }}$ are defined as maximum width and height of the tunnel, resp.). The heights of TX1 and RX1 are $6.5 \mathrm{~m}$ and $3 \mathrm{~m}$, respectively. Both TX1 and RX1 are located in the middle of the tunnel. The prior works show that totally 18 smooth surfaces which constitute the tunnel digital map can provide effective results and keep the computational complexity at reasonable level.

As depicted in Figure 3(c), the train is installed with two antennas in the head and the tail, respectively; the distance between two adjacent base stations (BSs) is $1 \mathrm{~km}$; the train is assumed to be $200 \mathrm{~m}$ long. As indicated in Figure 3(c), the tail antenna (RX1) communicates with the backward BS (TX1) while the head antenna (RX2) communicates with the forward BS (TX2). Note that, in this study, only the channel characteristics of Link 1 are investigated with the symmetric manner of Link 1 and Link 2. Furthermore, two radiation lobes of antennas in Figure 3(c) are used to illustrate the pointing directions of the directional antennas which are used in the TXs. Moreover, Figure 3(b) illustrates a snapshot in the simulation. In order to characterize the channel in this tunnel, the simulation scenario and system setups in this study follow the real requirements of the mobile communication system described in [15]. The scenario and system setups of the simulation are listed in Table 1.

3.2. Antenna Setups. As it is widely recognized that the directional antenna is indispensable for mmWave communication system, the detailed effects of directional antenna on mmWave channels still lack a careful investigation, especially for HSR channels. In other words, though the proper use of directional antenna gives a high compensation to received power, the distinctions of the radio channels characteristics between applying directional antenna and applying omnidirectional antenna are not clear. Therefore, in this study, three 


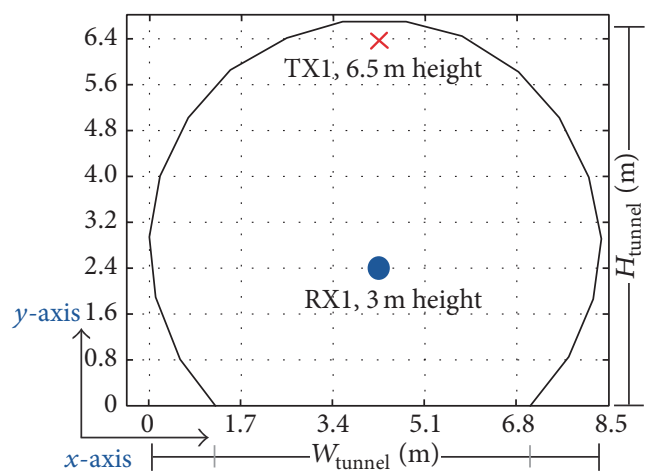

(a)

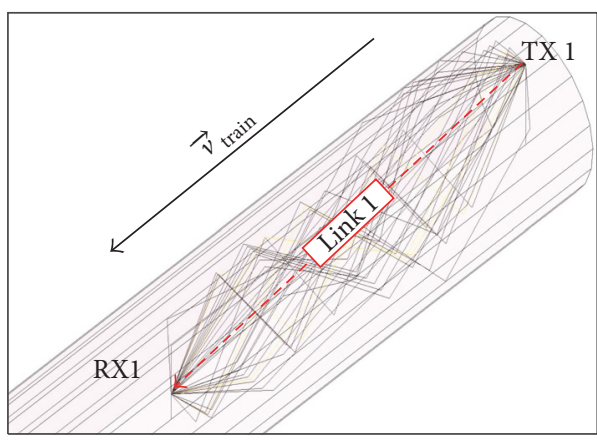

(b)

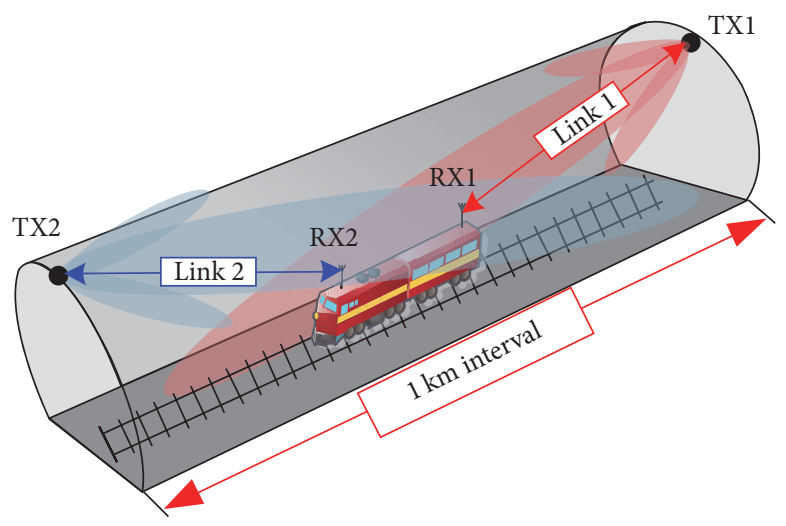

(c)

FIGURE 3: (a) Cross section of the tunnel in the simulation. (b) One snapshot of the simulation in the tunnel. (c) The sketch map for the scenario.

TABLE 1: Computation time ( $\mathrm{min})$ of total 1000 snapshots for orders of reflection.

\begin{tabular}{lccccccc}
\hline 1 st & 2nd & 3rd & 4th & 5 th & 6th & 7th & 8th \\
\hline $2.8(\mathrm{~s})$ & $5.7(\mathrm{~s})$ & $11.4(\mathrm{~s})$ & $36.1(\mathrm{~s})$ & $212.6(\mathrm{~s})$ & $1.5 \times 10^{3}(\mathrm{~s})$ & $1.1 \times 10^{4}(\mathrm{~s})$ & $8.4 \times 10^{4}(\mathrm{~s})$ \\
\hline
\end{tabular}

antenna setups are employed under various combinations of directional antenna and omnidirectional antenna; refer to Figure 4. They are defined as follows:

(1) Direc.-Direc.: TX1 and RX1 are both with the directional antennas. The antenna at TX1 is statically pointing along the tunnel while the antenna at RX1 is pointing at opposite direction of TX1 antenna.

(2) Direc.-Omni.: TX1 is with directional antenna and $\mathrm{RX} 1$ is with omnidirectional antenna. The antenna at TX1 is statically pointing along the tunnel.

(3) Omni.-Omni.: TX1 and RX1 are both with the omnidirectional antennas.

The directional antenna is designed by drawing sinusoidal chart in polar coordinate as horizontal and vertical patterns. The following studies will be carried out in describing radio channel characteristics of three antenna setups.

3.3. Number of Frequencies in Simulation. As the simulation configuration should follow the real mobile communication system described in [15], the simulation frequency bandwidth is set to be $125 \mathrm{MHz}$. Moreover, we want to expose the channel characteristics over a frequency range of $31.5 \mathrm{GHz} 33.5 \mathrm{GHz}$. So, the number of the center frequency points $N_{f}$ is chosen sufficiently enough. Therefore, total 264 center frequency points are considered in this study $[9,33,34]$.

3.4. Order of Multireflection in Simulation. The computational complex of RT simulation is significantly affected by multireflection which requires massive cyclic and traversal search of RT kernel. Although higher order of reflection greatly decreases the efficiency of RT simulations, the higher order of reflection gives more accurate simulation results. Therefore, the order of reflection should be selected very carefully. Before massive RT channel simulations, we studied the power contributions of each order of reflection in the same arched tunnel in Omni.-Omni. case. Figure 5 illustrates the percentages of overall power of each order of reflection compared to total received power. In the figure, on the whole, it is apparent that the overall received power which includes power contributions from LOS to 5 th order of reflection strikingly reaches $99 \%$ of total received power. Accordingly, 


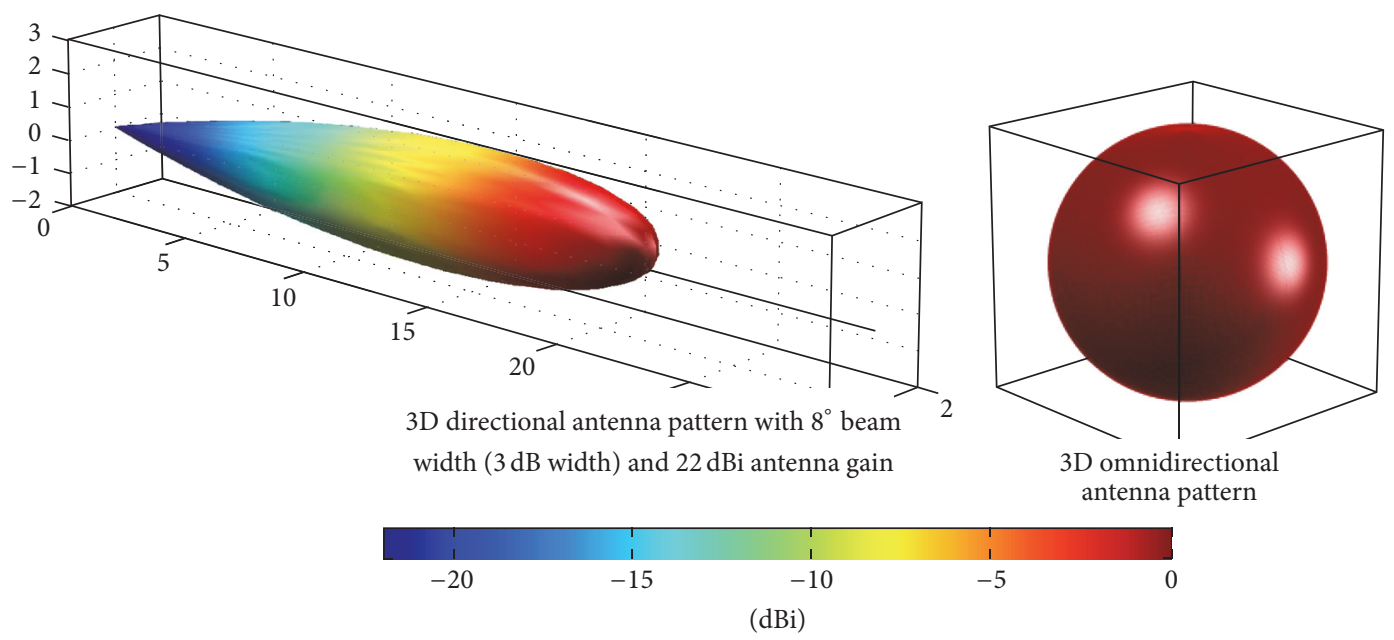

FIgURE 4: The directional antenna and omnidirectional antenna in this study.

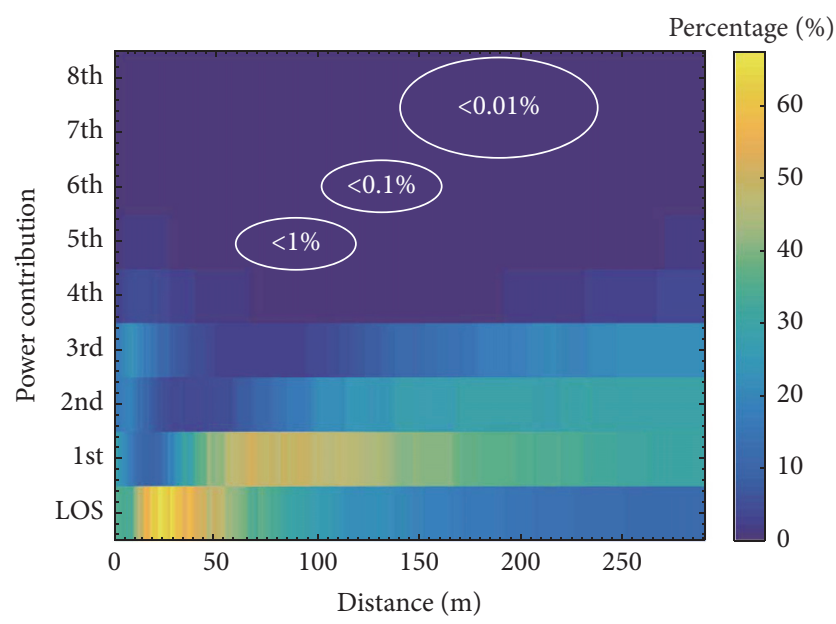

Figure 5: Percentages of overall power contribution of each reflection order with distance changes in Omni.-Omni. case.

we recorded the RT computation time of 1000 snapshots for considering up to $n$th order of reflection (cf. Table 1). As can be seen, the computation time is of exponential growth with the increase of reflection order. Comparing results of Figure 5 and Table 1, the percentages of power contribution of reflection orders higher than 6 th are less than $0.1 \%$ while they computation time are strikingly hundred times larger than that of lower reflection orders. Therefore, we limited maximum reflection order at 5 th which gives accurate simulation results while keeping the computation time acceptable.

Finally, Table 2 gives an overview of scenario setups and simulation configuration in this study.

\section{Study in System Feasibility and Partition for Regions}

4.1. System Feasibility with Different Antenna Setups. In wireless communication systems, coverage of the system is
TABLE 2: Scenario setups and simulation.

\begin{tabular}{|c|c|}
\hline Tunnel type [14] & Arched tunnel \\
\hline Antenna types & Directional antenna \\
\hline (Figure 4) & Omnidirectional antenna \\
\hline Material of tunnel & Sulphoaluminate cement \\
\hline Material permittivity & $\varepsilon_{r}=3.47-j 0.15$ \\
\hline Material scattering parameters & $S=0.00118, \alpha_{R}=120$ \\
\hline Tunnel length & $1 \mathrm{~km}$ \\
\hline Heights of TX and RX & $6.5 \mathrm{~m}$ and $3 \mathrm{~m}$ \\
\hline Speed of HSR & $360 \mathrm{~km} / \mathrm{h}$ \\
\hline System bandwidth for a link & $125 \mathrm{MHz}$ \\
\hline Frequency range investigated & $31.5 \mathrm{GHz} \sim 33.5 \mathrm{GHz}$ \\
\hline Transmit power & $30 \mathrm{dBm}[6]$ \\
\hline Cable loss & $6 \mathrm{~dB}$ \\
\hline Final channel sample interval & $2 \mathrm{~mm}$ \\
\hline
\end{tabular}

defined or controlled by a minimum required signal-noise ratio (SNR). The SNR is calculated by

$$
\operatorname{SNR}(\mathrm{dB})=P-\left(-174+10 \cdot \log _{10}(W)+N_{F}\right) .
$$

The value $P$ is the received power without small-scale fading which is excluded by averaging received signal with a $40 \lambda$ sliding/overlapped window [35]. $W$ is the channel simulation bandwidth; it is $125 \mathrm{MHz}$ in this study. $N_{F}$ is the noise figure which is the noise factor expressed in decibel. Here, $N_{F}$ is practically assumed to be $10 \mathrm{~dB}$. Further, the number -174 is widely used as spectral noise power density for $1 \mathrm{~Hz}$.

As shown in Figure 6, the channel performances of different antenna setups vary considerably in the near region (which is defined in Section 4.2). In Direc.-Direc. and Direc.Omni. cases, with the RX1 moving away from the TX1, the LOS component gradually enters the illumination of the main lobe ( $3 \mathrm{~dB}$ beam width) of the directional antenna. This process causes the SNR obvious increases in the distance ranging from $15 \mathrm{~m}$ to $50 \mathrm{~m}$. Afterwards, when RX1 moves into far region, the LOS and lots of NLOS components enter 


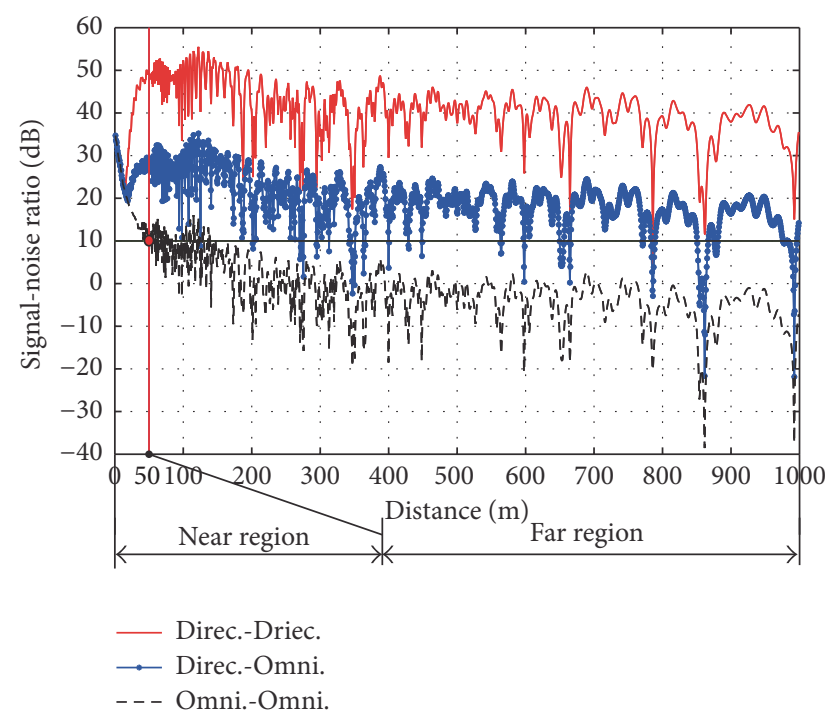

FIgURE 6: SNRs of different antenna setups. The red vertical solid line is the partition for two regions; the black horizontal line depicts the minimum threshold for the available SNR.

the illumination of the main lobe of the directional antenna. Obviously, the fluctuations of the SNRs of different antenna setups undergo a similar tendency in far region.

According to Figure 6, if the minimum SNR is $10 \mathrm{~dB}$ for a reliable detecting [6], that is, system minimum available threshold, this communication system in tunnel can support more than $1 \mathrm{~km}$ coverage range in the Direc.-Direc. and Direc.-Omni. cases. But, it is difficult to support $1 \mathrm{~km}$ signal coverage when deep shadow fading exists. In the Omni.Omni. case, the system can only support coverage range less than $50 \mathrm{~m}$. Although it is obvious that the directional antenna brings a better performance of signal coverage, the detailed channel characteristics are still under research.

4.2. Definition of Regions for Radio Channel Analysis. As it is shown in Figure 6, the received SNRs are obviously different in different regions. The reasons are mainly depending on the antennas used in simulation, for example, half-power bandwidth (HPBW), pointing direction, and position [36]. The following channel characteristics should be studied in different regions. Figure 7 gives the sketch of partition for regions. $H_{\mathrm{BS}}$ and $H_{\mathrm{Ant}}$ are the heights of BS (TX1) and RX1, respectively; $\theta$ and $\alpha$ are the inclination angle and HPBW of the directional antenna (TX1). The red solid line indicates the pointing direction of the TX1. The two black dotted lines depict the region which will be illuminated by the antenna main lobe. The value $D$ determines the boundary between near region and far region which can be calculated by [36]

$$
D=\frac{H_{\mathrm{BS}}-H_{\text {Ant. }}}{\tan (\theta+\alpha / 2)} .
$$

In this study, $\alpha=8^{\circ}, \theta=0^{\circ}, H_{\mathrm{BS}}=6.5 \mathrm{~m}$, and $H_{\text {Ant. }}=3 \mathrm{~m}$. The angle $(\theta+\alpha / 2)$ indicates the pointing direction plus half of the (elevation) HPBW. As a result, the length of $D$ in this study is $50 \mathrm{~m}$.

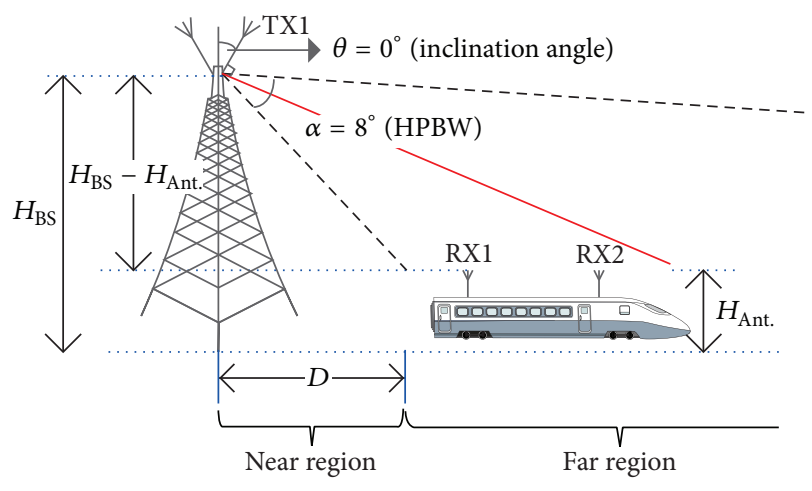

Figure 7: Sketch for region definition.

\section{The Detailed mmWave Channel Characteristics in HSR Tunnel Scenario}

Here, the radio channel characteristics will be presented in time, frequency, and polarization domains in order to help in designing a robust and sophisticated wireless communication system in HSR scenarios.

5.1. Path Loss and Shadow Fading Extraction. The largescale fading (including path loss and shadowing fading) are obtained by averaging received power with a 40 -wavelength window [35]. The large-scale fading is generally expressed as a log-distance path loss model $L(d)$ with a path loss exponent $(n)$ :

$$
L(d)=\bar{L}\left(d_{0}\right)+10 n \lg \left(\frac{d}{d_{0}}\right)+X_{\sigma},
$$

where $L(d)$ is the function of $d$ which indicates the distance between TX1 and RX1. $\bar{L}\left(d_{0}\right)$ is the intercept value at referenced distance $\left(d_{0}\right) . X_{\sigma}$ is the shadow fading. Figure 8 gives one example of extraction process of $n$ at a frequency center of $32.5 \mathrm{GHz}$ in far region. Note that the following analyses for path loss and shadow fading are mainly in far region. This is because, in the near region, the fluctuation of the received power is largely dominated by antenna radiation pattern.

In Figure 8, a red solid line indicates the least square fitting result of the simulated data (marked in blue). The path loss exponent is the slope of the red solid line. It is around 1.1 in far region, which indicates the small attenuation of wave propagation in tunnel. This character may stem from the waveguide effects caused by tubular structure of the tunnel where the reflection attenuation will be small enough when incident angles of reflected rays are quite large in far region. The path loss exponents of different antenna setups at frequencies in the range $31.5 \mathrm{GHz} 33.5 \mathrm{GHz}$ are calculated and shown with statistical values in Table 3.

5.2. Amplitude Distribution of Shadow Fading. As expressed in (6), the shading fading $X_{\sigma}$ can be extracted from the large-scale fading $L(d) . X_{\sigma}$ is conventionally modeled as a log-normal distribution $[14,36]$ which is confirmed in this study. 
TABLE 3: Channel fading characteristics statistics.

\begin{tabular}{|c|c|c|c|c|c|c|}
\hline \multirow[t]{2}{*}{ Setups } & \multicolumn{2}{|c|}{ Direc.-Direc. } & \multicolumn{2}{|c|}{ Direc.-Omni. } & \multicolumn{2}{|c|}{ Omni.-Omni. } \\
\hline & \multicolumn{4}{|c|}{ Path loss exponent $(n)$} & \\
\hline Min & \multicolumn{2}{|c|}{1.06} & \multicolumn{2}{|c|}{1.06} & \multicolumn{2}{|c|}{1.07} \\
\hline Mean & \multicolumn{2}{|c|}{1.10} & \multicolumn{2}{|c|}{1.10} & \multicolumn{2}{|c|}{1.11} \\
\hline Max & \multicolumn{2}{|c|}{1.13} & \multicolumn{2}{|c|}{1.13} & \multicolumn{2}{|c|}{1.13} \\
\hline \multicolumn{7}{|c|}{ Shadow fading standard deviation $(\sigma)[\mathrm{dB}]$} \\
\hline Min & \multicolumn{2}{|c|}{3.32} & \multicolumn{2}{|c|}{3.39} & \multicolumn{2}{|c|}{3.42} \\
\hline Mean & \multicolumn{2}{|c|}{3.43} & \multicolumn{2}{|c|}{3.49} & \multicolumn{2}{|c|}{3.47} \\
\hline $\operatorname{Max}$ & \multicolumn{2}{|c|}{3.49} & & & & \\
\hline & & & tion dis & & & \\
\hline$d_{\text {cor }}$ & $d$ & $d$ & $d$ & $d$ & $d$ & $d$ \\
\hline$[m]$ & $(0.5)$ & $\left(e^{-1}\right)$ & $(0.5)$ & $\left(e^{-1}\right)$ & $(0.5)$ & $\left(e^{-1}\right)$ \\
\hline $10 \%$ & 1.20 & 1.60 & 0.90 & 1.40 & 0.80 & 1.00 \\
\hline $50 \%$ & 2.03 & 2.75 & 1.89 & 2.60 & 1.73 & 2.30 \\
\hline $90 \%$ & 3.81 & 5.01 & 3.41 & 4.70 & 3.34 & 4.31 \\
\hline Mean & 2.46 & 3.36 & 2.08 & 3.09 & 1.89 & 2.68 \\
\hline & & & $K$-fact & & & \\
\hline Regions & Near & Far & Near & Far & Near & Far \\
\hline $10 \%$ & 5.90 & -7.79 & 5.78 & -8.28 & 3.75 & -8.29 \\
\hline $50 \%$ & 19.48 & -6.98 & 10.59 & -7.47 & 7.28 & -7.48 \\
\hline $90 \%$ & 36.57 & -2.29 & 20.85 & -3.60 & 12.62 & -4.64 \\
\hline Mean & 20.31 & -5.80 & 12.28 & -6.54 & 7.80 & -6.85 \\
\hline & & & uare de & & & \\
\hline Regions & Near & Far & Near & Far & Near & Far \\
\hline $10 \%$ & 0.11 & 0.40 & 0.67 & 0.44 & 2.62 & 0.44 \\
\hline $50 \%$ & 0.32 & 0.47 & 1.94 & 0.52 & 4.82 & 0.58 \\
\hline $90 \%$ & 5.72 & 0.54 & 5.72 & 0.69 & 5.72 & 1.20 \\
\hline Mean & 2.12 & 0.47 & 2.97 & 0.54 & 4.42 & 0.70 \\
\hline & & & re Dopp & & & \\
\hline Regions & Near & Far & Near & Far & Near & Far \\
\hline $10 \%$ & 0.40 & 0.40 & 0.41 & 0.40 & 0.50 & 0.40 \\
\hline $50 \%$ & 0.42 & 0.41 & 0.55 & 0.41 & 0.94 & 0.41 \\
\hline $90 \%$ & 2.46 & 0.42 & 2.46 & 0.42 & 2.45 & 0.42 \\
\hline Mean & 0.95 & 0.41 & 1.04 & 0.41 & 1.20 & 0.41 \\
\hline & & & $\mathrm{PD}_{\theta}[\mathrm{d}]$ & & & \\
\hline Regions & Near & Far & Near & Far & Near & Far \\
\hline $10 \%$ & 10.44 & -5.80 & 8.07 & -6.47 & 5.44 & -6.61 \\
\hline $50 \%$ & 28.45 & 3.00 & 18.72 & 2.20 & 14.17 & 2.26 \\
\hline $90 \%$ & 41.11 & 12.93 & 29.03 & 12.63 & 23.45 & 12.77 \\
\hline Mean & 26.84 & 3.13 & 18.66 & 2.54 & 14.34 & 2.49 \\
\hline & & & $\mathrm{PD}_{\varphi}[\mathrm{d}]$ & & & \\
\hline Regions & Near & Far & Near & Far & Near & Far \\
\hline $10 \%$ & 10.52 & -7.99 & 8.28 & -8.99 & 5.41 & -8.98 \\
\hline $50 \%$ & 28.43 & 1.66 & 18.21 & 1.23 & 11.53 & 0.90 \\
\hline $90 \%$ & 41.21 & 10.81 & 28.52 & 10.43 & 20.10 & 10.11 \\
\hline Mean & 26.90 & 1.38 & 18.33 & 0.80 & 12.23 & 0.63 \\
\hline
\end{tabular}

Figure 9 describes the probability density function (PDF) of shadowing fading in Direc.-Direc. case at $32.5 \mathrm{GHz}$ with the results of $\mu=-1.7052 \mathrm{~dB}$ and $\sigma=3.4443 \mathrm{~dB}$, although, as the shadowing fading was extracted by (6), some deep fading inevitably leads to a no-zero value of $\mu$. However, the modeled $\sigma$ is still valuable for studying the channel shadowing characteristics in tunnel. The statistic value of $\sigma$ at whole frequencies can be found in Table 3. 


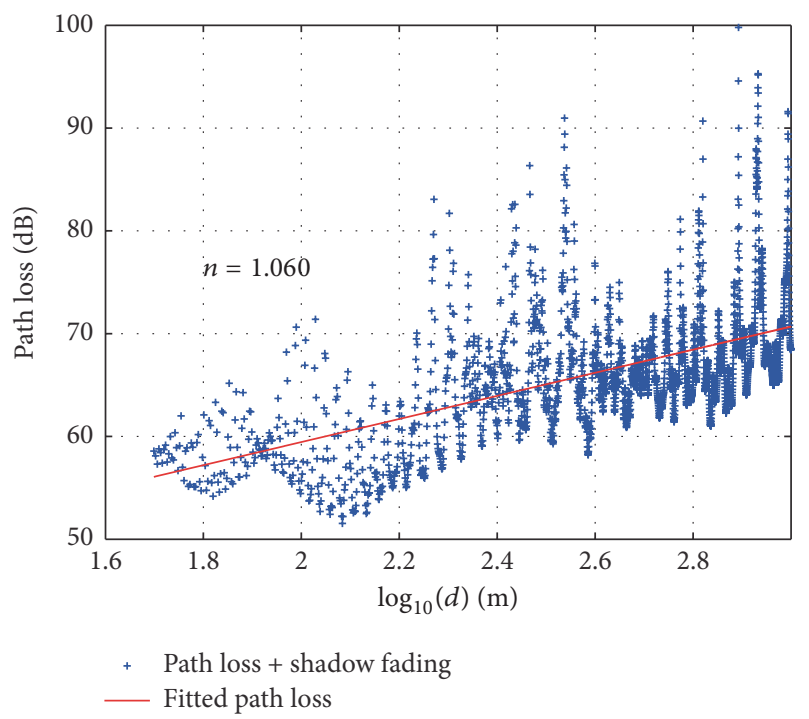

Figure 8: Path loss fitting at $32.5 \mathrm{GHz}$ in Direc.-Direc. case in far region.

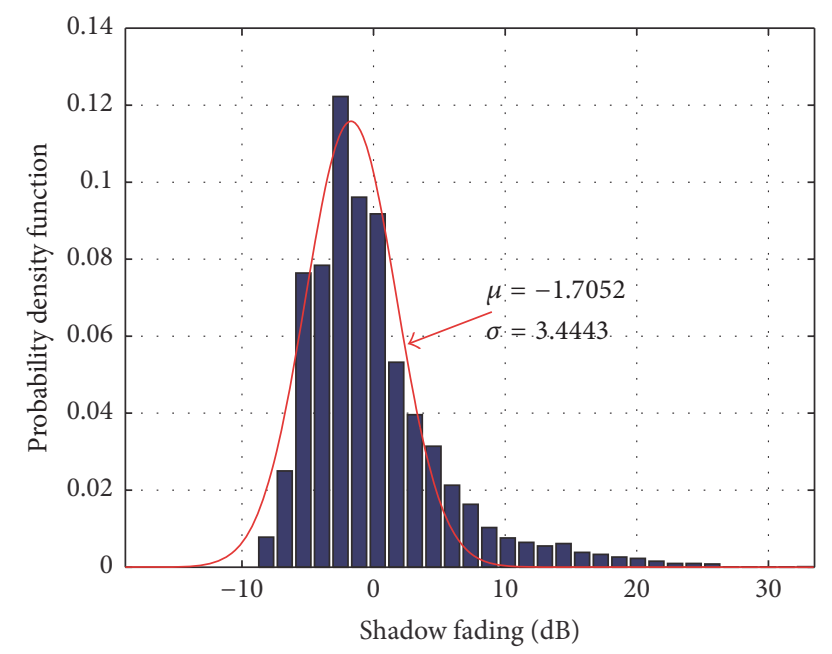

Simulation

— Fitted normal distribution

FIGURE 9: Fitting for amplitude distribution of shadow fading.

5.2.1. Autocorrelation of Shadow Fading. As shadowing causes the channel deep fading, the communication links tend to be interrupted (refer to Figure 6). To overcome the potential communication interruption, the autocorrelation of shadow fading should be well-studied. The autocorrelation coefficient of shadow fading is one important characteristic for designing distributed antenna system, which is defined as

$$
\rho_{1,2}=\frac{E\left\{S\left(d_{1}\right) S\left(d_{2}\right)\right\}}{\sigma\left(d_{1}\right) \sigma\left(d_{2}\right)},
$$

where $E\{\cdot\}$ denotes the expectation; $S(d)$ is the expression of the shadow fading at distance $d ; \sigma(d)$ is the expression of the standard deviation for the shadow fading at distance $d$.

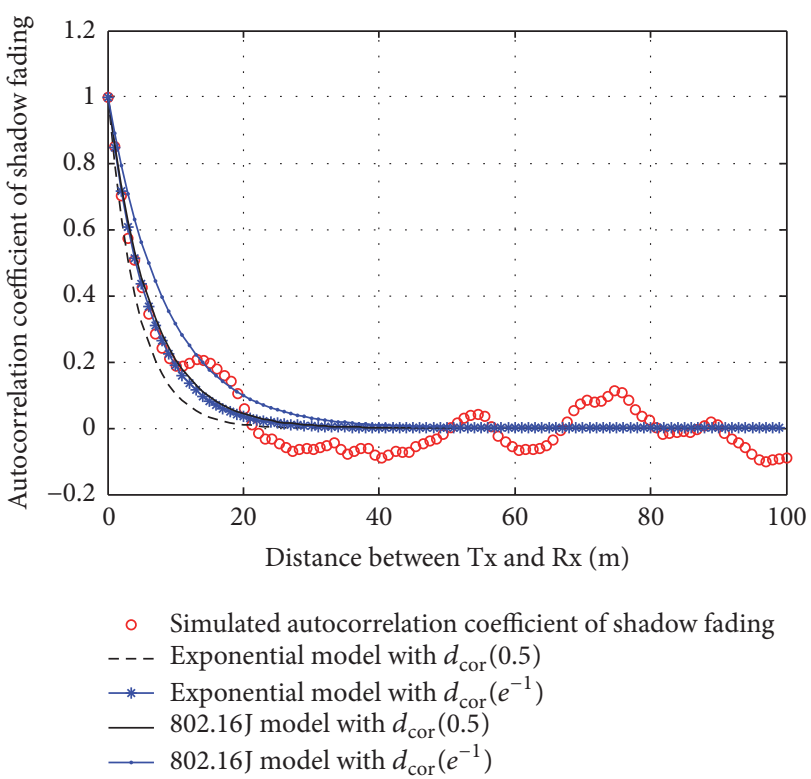

FIgURE 10: Autocorrelation coefficient of shadow fading in Direc.Direc. case at $32.5 \mathrm{GHz}$ in far region.

Further, two widely used empirical models are employed to fit the autocorrelation coefficient [14]: the exponential model and 802.16J model. The former is accepted in WINNER II model:

$$
\rho_{\exp }(\Delta d)=e^{\left(-\Delta d / d_{\text {cor }}\right)}
$$

the latter is presented in standard IEEE 802.16J:

$$
\rho_{\exp }(\Delta d)=e^{\left(-\Delta d / d_{\text {cor }}\right) \cdot \ln 2}
$$

In (8) and (9), $\Delta d$ is the distance between two interested positions $\left(d_{1}\right.$ and $\left.d_{2}\right)$. There are mainly two definitions for decorrelation distance $d_{\text {cor }}: d_{\text {cor }}(0.5)$ and $d_{\text {cor }}\left(e^{-1}\right)$. They present the correlation coefficient equal to thresholds 0.5 and $e^{-1}$, respectively [14]. Obviously, these two models have same structure. Figure 10 gives the autocorrelation coefficient of shadow fading in Direc.-Direc. case at $32.5 \mathrm{GHz}$ in far region. In conclusion, 802.16 J model is fitting well when threshold is 0.5. The exponential model performs better when threshold is $e^{-1}$.

5.2.2. Decorrelation Distance of Shadow Fading. Compensating the deep shadowing fading of the channel is generally used in multiantennas technology. Therefore, antennas should be separated long enough to obtain channel diversity gain. This distance is so-called decorrelation distance. The decorrelation distances for each RX1 position along the tunnel is extracted by using (7) with two thresholds $\left(0.5\right.$ and $\left.e^{-1}\right)$ at whole frequencies in the range $31.5 \mathrm{GHz}$ $33.5 \mathrm{GHz}$. Figure 11(a) is an example of decorrelation distance at $32.5 \mathrm{GHz}$ in far region. Obviously, there are rare differences among decorrelation distances of different antenna setups. Around a distance of $150 \mathrm{~m}$, the Direc.-Direc. case reveals a longer decorrelation distance as shown in Figure 11(a). For 


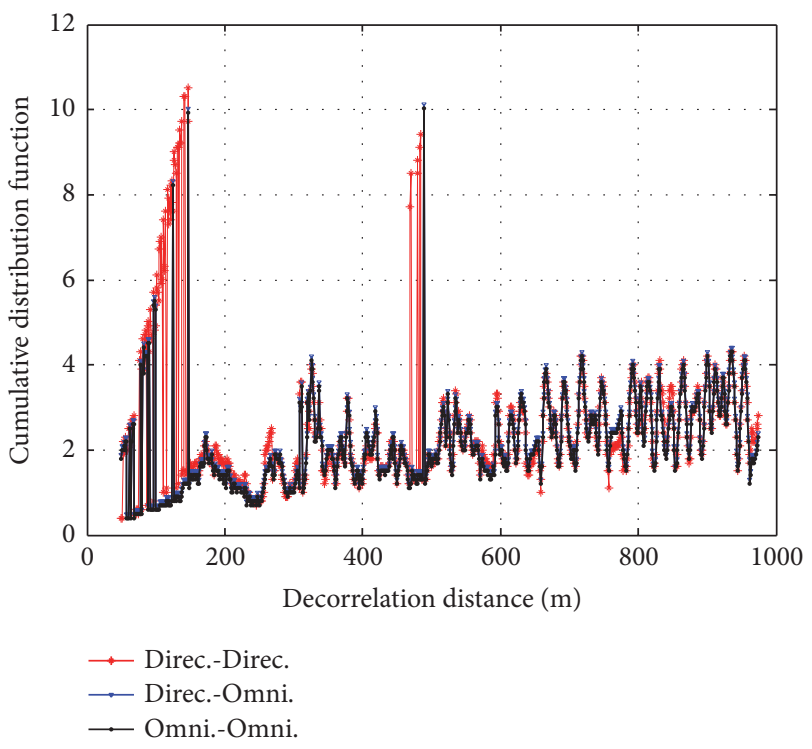

(a)
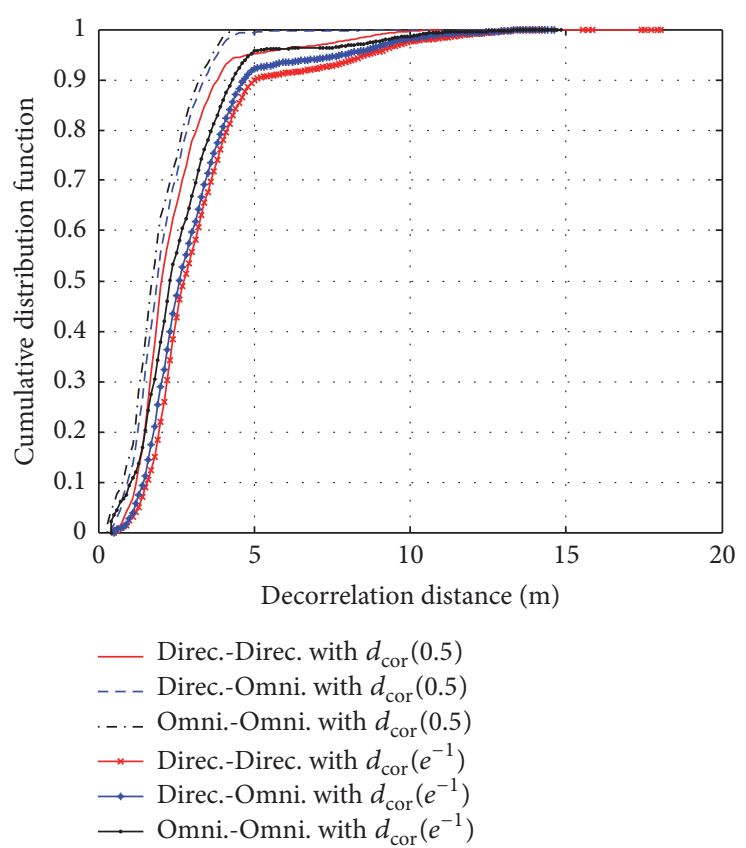

(b)

Figure 11: (a) Decorrelation distances of three antenna setups at $32.5 \mathrm{GHz}$ in far region. (b) For three antenna setups, the figure shows the $\mathrm{CDF}$ of the decorrelation distances at whole frequencies with thresholds 0.5 and $e^{-1}$ in far region.

the whole frequency range, Figure 11(b) describes cumulative distribution function (CDF) of the decorrelation distances of three antenna setups. Furthermore, Table 3 lists their statistic values.

For all three antenna setups, the decorrelation distances calculated by using threshold $e^{-1}$ are no doubt longer than that using threshold 0.5. Moreover, Direc.-Direc. performs a longer decorrelation distance value (mean value around 2.5) than other cases (mean value around $2.3 \mathrm{~m}$ and $2 \mathrm{~m}$, resp.). These indicate that the decorrelation distance will become longer when directional antenna is employed.

5.3. Rician K-Factor for the Received Signal. The time-varying fading characteristic of the signal is normally modeled by Rician $K$-factor when LOS component exists [37]. the Rician $K$-factor is defined as the ratio of the power of LOS component to the total power of NLOS components.

The Rician $K$-factor at $32.5 \mathrm{GHz}$ is shown in Figure 12 (a). In the near region, the $K$-factor experiences a rapid change. In Omni.-Omni. case, this process can be described by the narrow structure of the tunnel which causes the attenuation of reflected components. For other two cases, this rapid change mainly stems from the radiation pattern of directional antenna. In the far region, the $K$-factors of all three antenna setups decrease slowly. Moreover, Figure 12(b) gives the CDF of three antenna setups at whole frequencies in the range $31.5 \mathrm{GHz} 33.5 \mathrm{GHz}$ in both near region and far region. It is obvious that, in the near region, the $K$-factor varies intensely. But, in the far region, the differences among three $K$-factors are fairly small. The statistical values are listed in Table 3 where we find that the $K$-factor (in $\mathrm{dB}$ ) is positive in near region but is negative in the far region. This characteristic indicates that the dominant power contribution of the received signal is changing from the LOS component to the NLOS components.

5.4. Delay Characteristics in the Tunnel. The root mean square (RMS) delay spread is widely known as the single parameter that can provide a quick overview of channel delay characteristics. It is defined as the normalized second-order moment of the power delay profile (PDP) which characterizes channel delay dispersion [37]. In this study, the RMS delay spread is calculated as follows:

$$
S_{\tau}(t)=\sqrt{\frac{\sum_{k=1}^{N(t)} P_{k}(t) \cdot \tau_{k}(t)^{2}}{\sum_{k=1}^{N(t)} P_{k}(t)}-\left(\frac{\sum_{k=1}^{N(t)} P_{k} \cdot \tau_{k}(t)}{\sum_{k=1}^{N(t)} P_{k}(t)}\right)^{2}},
$$

where $S_{\tau}(t)$ is the RMS delay spread; $P_{k}(t)$ is the power of $k$ th ray. As all the rays are specific with certain delay, power, and angle information, (10) is efficient for calculating the RMS delay spread directly from rays of RT kernel results.

Figure 14 depicts CDFs of the RMS delay spreads which were extracted in every snapshot at whole simulation frequencies. Apparently, when directional antennas are used in the system, the RMS delay spread will be decreased especially in near region. These results are in line with the phenomenon displayed in Figure 13 which specially compares PDPs of three antenna setups in near region. It is clear that the directional antenna can be a great spatial filter in near region that attenuates multipath components which are not illuminated by the main lobe of directional antenna. Therefore, when directional antennas are employed at both TX1 and RX1, the 


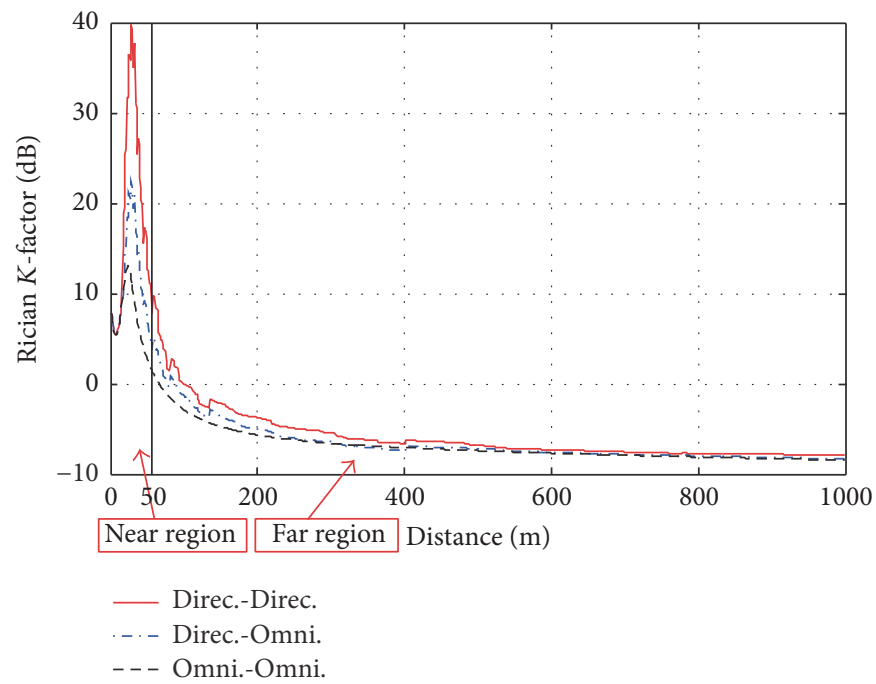

(a)

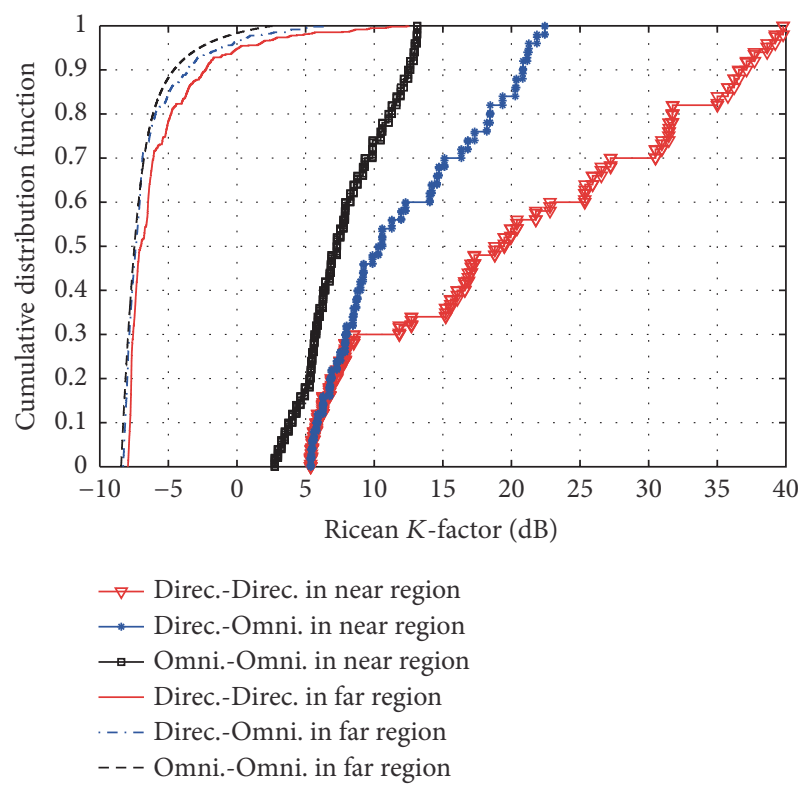

(b)

Figure 12: (a) Rician $K$-factor for different antenna setups at $32.5 \mathrm{GHz}$. (b) CDF of Rician $K$-factor in different antenna setups and regions at whole frequencies.

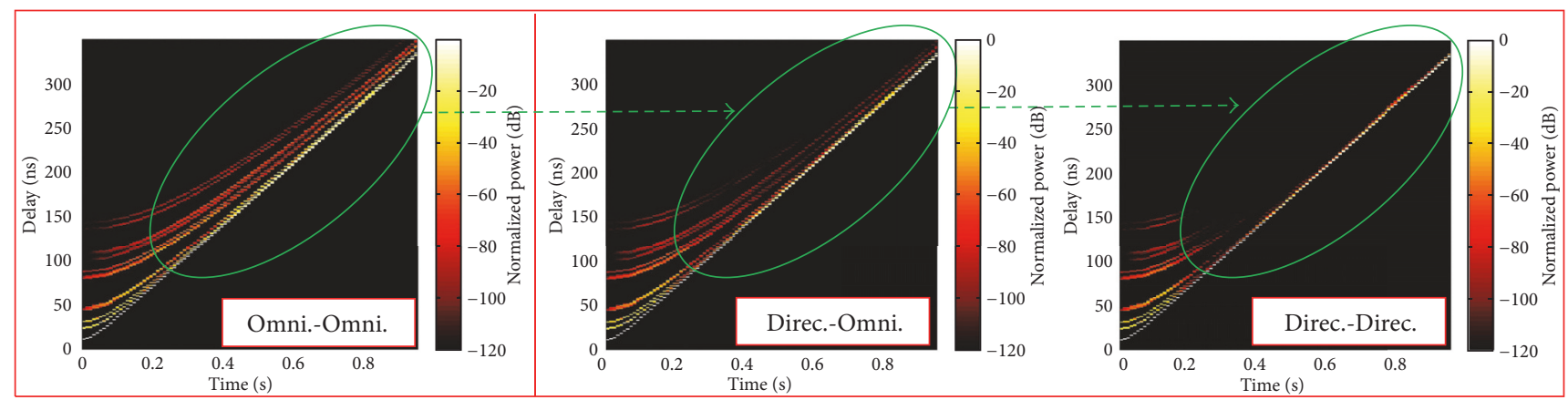

FIGURE 13: Partial enlarged views to describe the changing of the PDPs with different antenna setups in near region at 32.5 GHz.

minimum value of RMS delay spread can be obtained which is around $2.12 \mathrm{~ns}$ in near region and 0.47 in far region.

5.5. Doppler Characteristic in the Tunnel. As discussed previously, the HSR channels in tunnel were simulated at speed of $360 \mathrm{~km} / \mathrm{h}$. Therefore, the Doppler effect on the channels is widely of interest as it gives physical interpretation of the frequency shift caused by movement [37]. As can be seen in Figure 15, in near region, the train movement obviously spreads the Doppler spectrum in Omni.-Omni. case; but the spectrum shows a stable Doppler frequency shift with limited frequency spread in far region. The striking variations of the Doppler spectrum in near region are partly due to the fact that the incident angles of received rays are sparse and change rapidly, whereas, in the far region, the incident angles of received rays are very close and change slowly (referring to the tunnel narrow structure), which leads to a stable
Doppler frequency. Meanwhile, since the directional antenna attenuates lots of rays in near region, Figure 16 illustrates the detailed effects of directional antenna on Doppler spectra where some distinct differences among Doppler spectra are clearly shown. To better evaluate the Doppler effects, the CDFs of mean Doppler shifts and RMS Doppler spreads of three antenna setups are studied at whole frequencies in Figure 17. These two parameters are the moments of the Doppler spectra which can be calculated similar to the moments of the PDP [37]. According to Figure 17 and the statistic values listed in Table 3 , the same conclusion as that from Figure 16 can be obtained that directional antenna is like a spatial filter which causes larger mean Doppler shift and lower RMS Doppler spread in HSR tunnel.

Generally, the Doppler effects are studied with other second-order fading statistics that also closely related to channel dynamic characteristics and the quality of received 


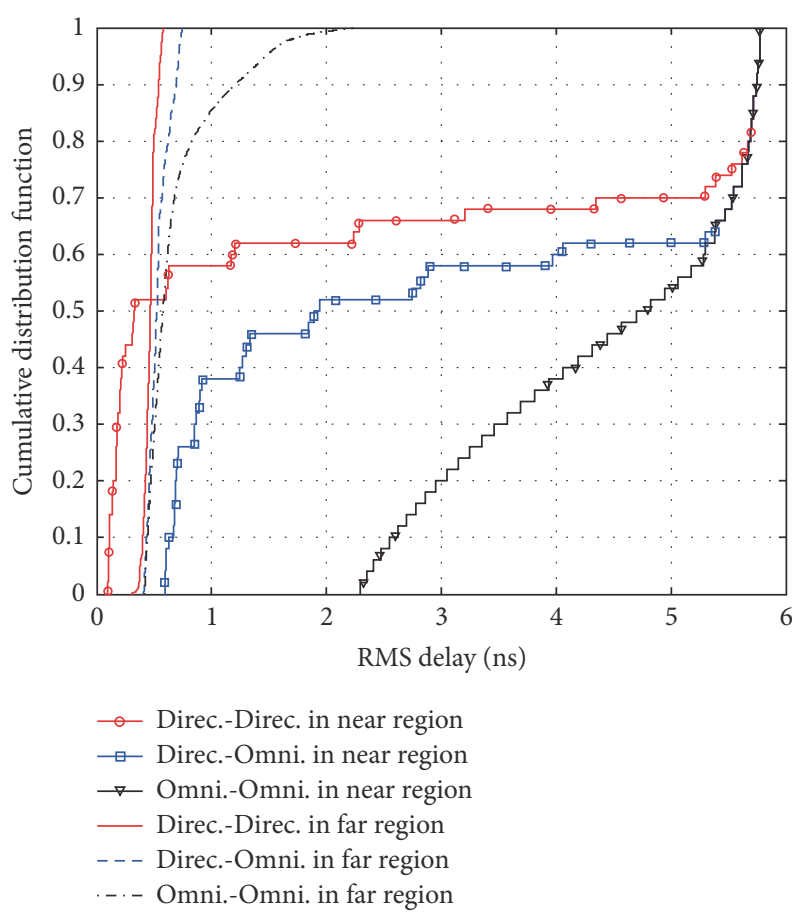

Figure 14: CDF of RMS delay in different antenna setups and regions at whole frequencies.

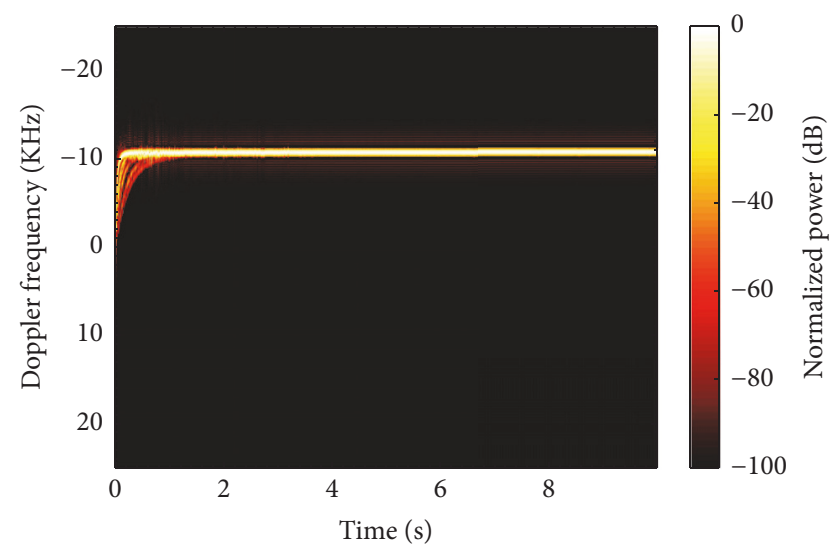

FIGURE 15: Doppler spectra of Omni.-Omni. case at $32.5 \mathrm{GHz}$ in tunnel.

signals. They are level cross rate (LCR) and average fade duration (AFD) [38]. As detailed in Figure 18(a), curves that stand for LCRs in far region exhibit lower LCRs compared to that in near region; and the result of Direc.-Direc. case in far region shows the minimum LCR. These phenomena are in line with observation results in Doppler spectra (cf. Figures 16 and 17). Additionally, since the LCRs of far region are far lower than LCRs of near region, the Doppler frequencies in far region are accordingly more stable with minimum Doppler spread and mean shift. However, although the AFDs exhibited in Figure 18(b) have no clear distinctions between different regions, the AFD of Direc.-Direc. case still shows the minimum value. And AFDs of three antenna setups in far region increase sharply when thresholds for AFD calculation are around $0 \mathrm{~dB}$, which indicate weaker fastfading characteristics of the channels in far region as well as weaker Doppler spread.

5.6. Polarization Characteristics in the Tunnel. The above analyses of channel characteristics describe the vertical polarization only. However, the channel polarization characteristics in the tunnel are valuable for system design. The radiated signal from a vertically polarized antenna will experience interactions that result in horizontal polarized component before it arrives at RX and vice versa [37]. This process is so-called depolarization process that has been depicted by a $2 \times 2$ matrix which is formulated by $P_{k}(t)$. Then, cross-polarization discriminations (XPD including $\mathrm{XPD}_{\theta}$ and $\mathrm{XPD}_{\varphi}$ ) are invited, which indicates leakage from one polarization to another by depolarization effect $[39,40]$ :

$$
\begin{aligned}
\mathrm{XPD}_{\theta} & =\frac{\left|h_{\mathrm{VV}}\right|^{2}}{\left|h_{\mathrm{VH}}\right|^{2}}, \\
\mathrm{XPD}_{\varphi} & =\frac{\left|h_{\mathrm{HH}}\right|^{2}}{\left|h_{\mathrm{HV}}\right|^{2}},
\end{aligned}
$$

where $\left|h_{\mathrm{VH}}\right|^{2}$ refers to the power transmitted in vertical polarization and received in horizontal polarization. $\left|h_{\mathrm{VV}}\right|^{2}$, $\left|h_{\mathrm{HH}}\right|^{2}$, and $\left|h_{\mathrm{HV}}\right|^{2}$ are defined accordingly.

Figure 19(a) illustrates $\mathrm{XPD}_{\theta}$ and $\mathrm{XPD}_{\varphi}$ simulated data in Direc.-Direc. case accompanying fitting lines. The depolarization processes of the channel are found to be similar for both vertical and horizontal polarized signal at $32.5 \mathrm{GHz}$. The tubular structure of tunnel which results in the depolarization process of the channel does not obviously prefer vertical or horizontal polarized signal. Moreover, the values of XPDs in near region are much larger than values in far region. As LOS component cannot be depolarized and gradually illuminated by antenna main lobe in near region, the numerator of (11) will increase with attached antenna gain to the LOS component. This causes an apparent change as shown in Figure 19(a).

Figures 19(b) and 19(c) show the CDFs of $\mathrm{XPD}_{\theta}$ and $\mathrm{XPD}_{\varphi}$ of three antenna setups at whole frequencies in different regions. We learn that using directional antenna can baffle depolarization process of the channel in near region. The statistic values of the results of depolarization process in the tunnel can be found in Table 3 .

5.7. Relative Discussions. Based on the radio channel characteristics and aforementioned analyses, Table 3 lists all the statistic values of the parameters. Then, the following discussions can be made:

(1) Path loss: the path loss exponents for each antenna setup are all around 1.1 in far region. The tubular and narrow structure of the tunnel probably causes the waveguide propagations which compensate the received power in far region. 


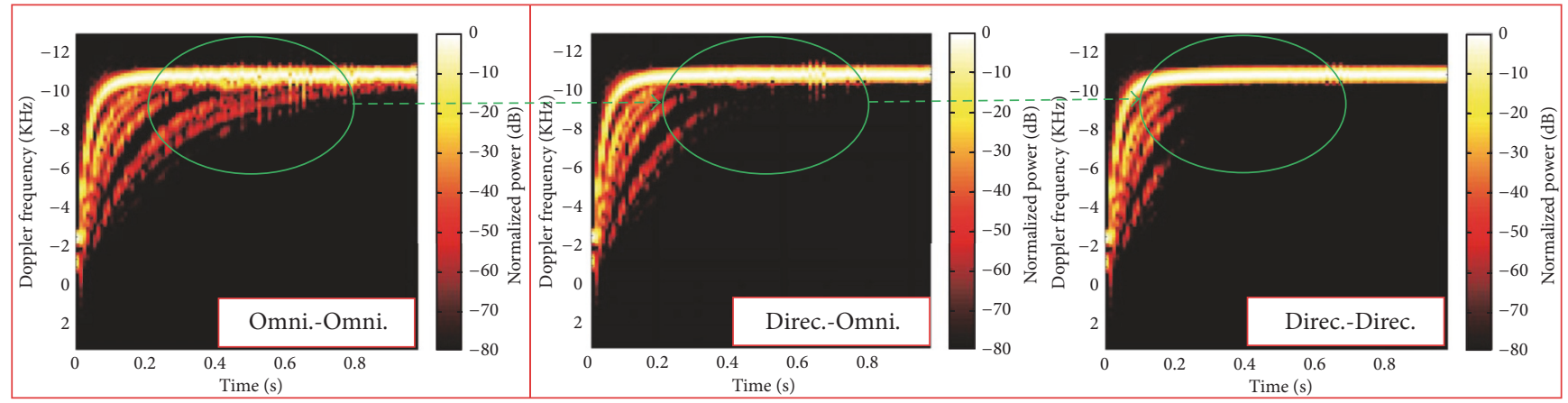

Figure 16: Partial enlarged views to describe the changing of the Doppler spectra with different antenna setups in near region at $32.5 \mathrm{GHz}$.

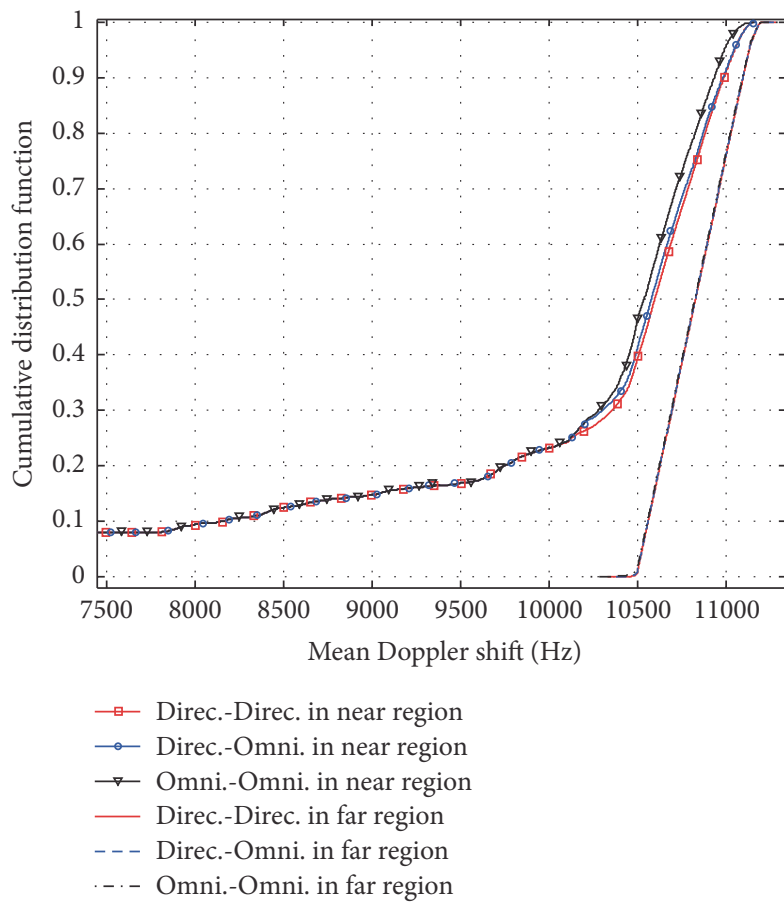

(a)

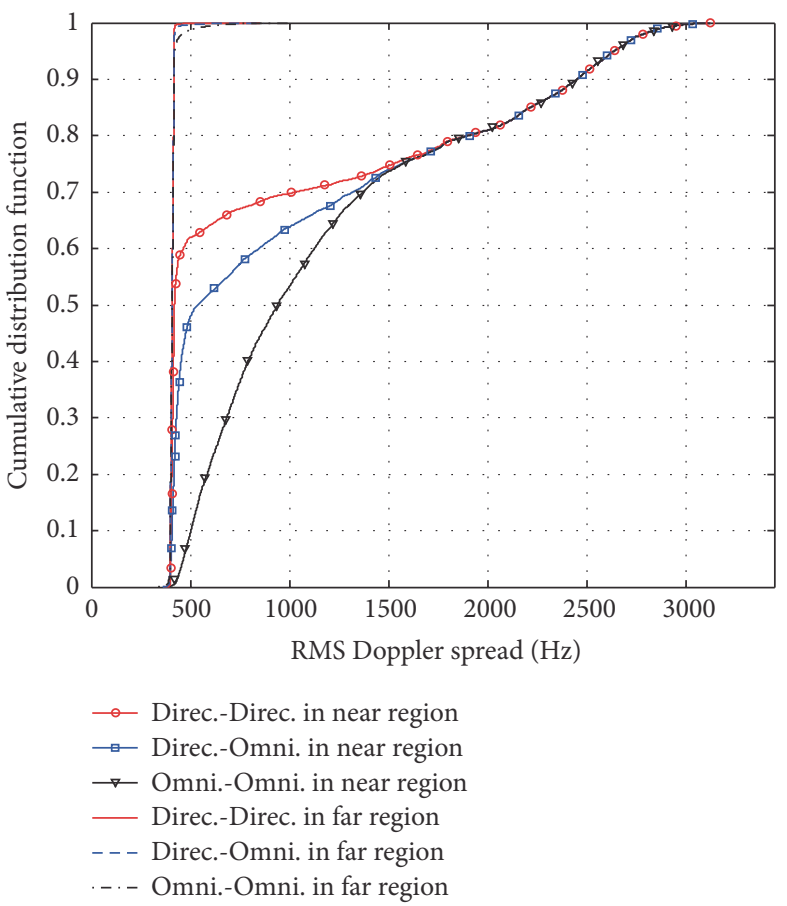

(b)

FIGURE 17: (a) CDFs of mean Doppler shift in different antenna setups and regions at whole frequencies. (b) CDFs of RMS Doppler spread in different antenna setups and regions at whole frequencies.

(2) Shadow fading: as the log-normal distribution can fit the shadow fading well, the shadow fading standard deviations for three antenna setups are similar and around $3.4 \mathrm{~dB}$ in the far region. This number is close to the measurement results in the tunnel at $5.7 \mathrm{GHz}$ [14].

(3) Decorrelation distance: the deep fading caused by shadow fading will decrease received power and decay system capacity. Also, possibly, it may result in pingpong effect when system is in handover. The decorrelation distances calculated by threshold $e^{-1}$ are obviously larger than that using threshold 0.5 . The mean value for Direc.-Direc. case is around $2.5 \mathrm{~m}$ (threshold
0.5) which is larger than other cases. In conclusion, as per directional antenna employed in system, the decorrelation distance increases $0.2-0.4 \mathrm{~m}$.

(4) Rician $K$-factor: the $K$-factors in two regions show great differences, especially for Direc.-Direc. case where the difference between the mean values of two regions is around $25 \mathrm{~dB}$. Even if the $K$-factors have a great variation in different regions, the $K$-factors of different antenna setups have little differences in far region (around $-6 \mathrm{~dB}$ ).

(5) RMS delay spread: the RMS delay spread will be deceased when directional antenna is employed. In the near region, the directional antenna performs as 


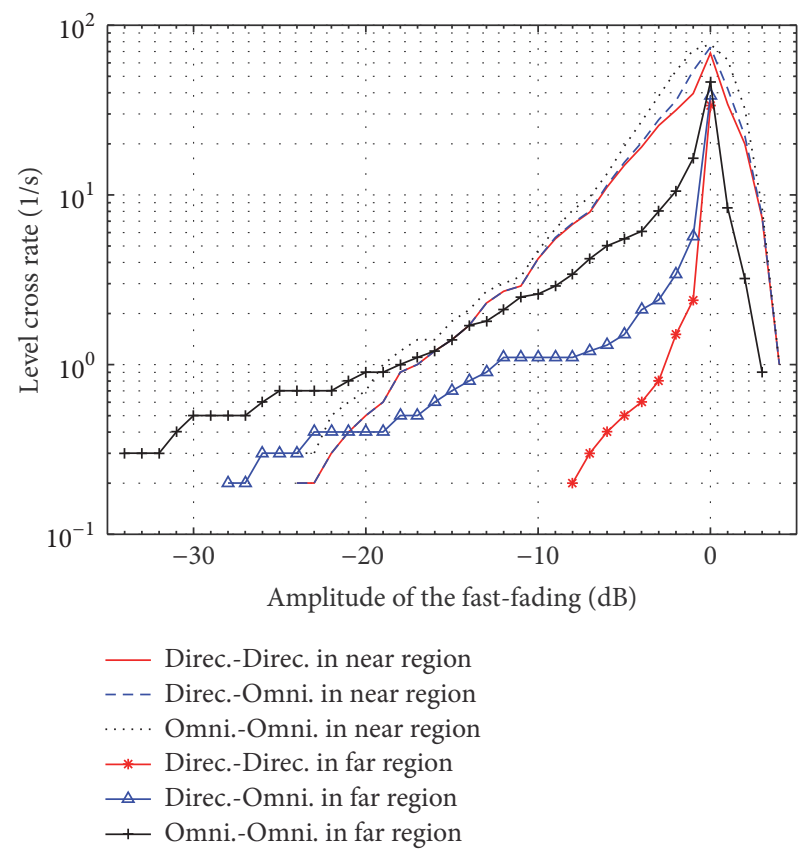

(a)

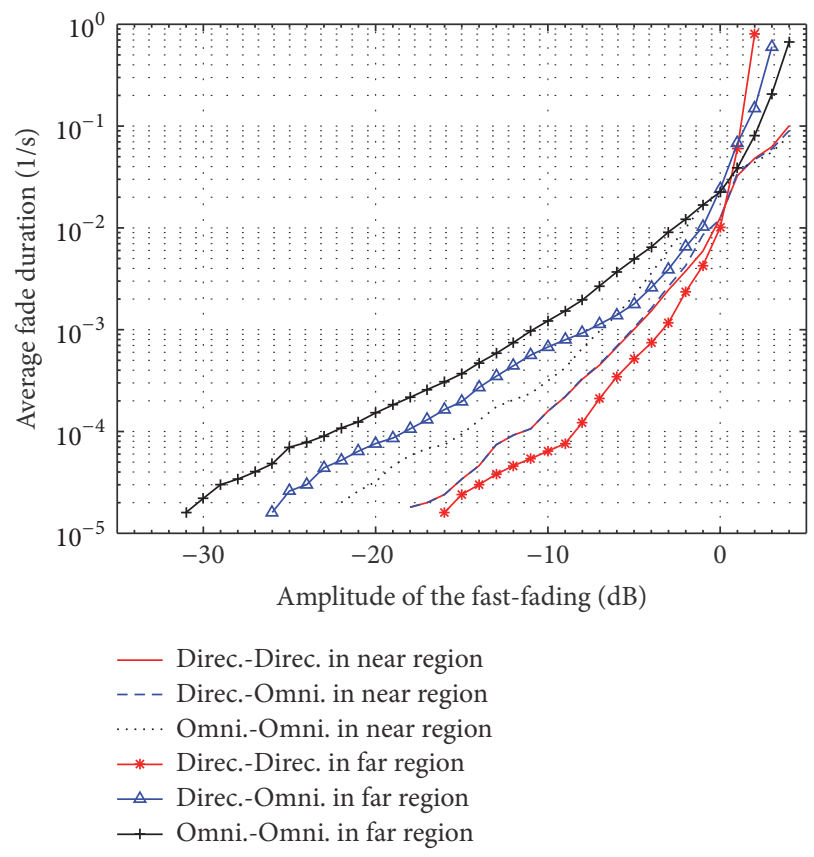

(b)

FIGURE 18: (a) Results of level cross rates of three antenna setups at $32.5 \mathrm{GHz}$. (b) Results of average fade durations of three antenna setups at $32.5 \mathrm{GHz}$.

a spatial filter which reduces the RMS delay spread by $1-1.5 \mathrm{~ns}$. In the far region, RMS delay spreads of different antenna setups are less than $1 \mathrm{~ns}$, and in the Direc.-Direc. case, the number will be less than $0.5 \mathrm{~ns}$.

(6) RMS Doppler spread: there are less than $100 \mathrm{~Hz}$ variations in RMS Doppler spreads when one or two directional antennas are employed in the system. All RMS Doppler spreads in far region are around $0.41 \mathrm{kHz}$. Furthermore, the RMS Doppler spread may be less influenced than RMS delay spread when directional antennas are employed.

(7) XPDs: for Direc.-Direc. and Direc.-Omni. cases, it is interesting to find that the mean values of $\mathrm{XPD}_{\theta}$ and $\mathrm{XPD}_{\varphi}$ are very close in near region and have a variation of $1.7 \mathrm{~dB}$ in far region. For Omni.-Omni. case, the values of $\mathrm{XPD}_{\theta}$ are $2 \mathrm{~dB}$ larger than $\mathrm{XPD}_{\varphi}$ in both the two regions. Since the vertical polarized signal shows an advantage in resisting depolarization compared to horizontal polarized signal in the tunnel, it is better to deploy vertical polarized antennas in the arched tunnel.

\section{Conclusion}

In this paper, $30 \mathrm{GHz}$ band HSR radio channels in an arched tunnel with different antenna setups are investigated by a RT tool. To enhance RT for more accurate simulations, a special material measurement campaign is performed for the cement which is the main construction material of the tunnel. Then, an advanced time-interpolation method is described for extracting small-scale channel characteristics. These two steps work as a promising and solid base for our extensive channel simulation at $31.5 \mathrm{GHz} 33.5 \mathrm{GHz}$. The channel characteristics are found to be widely different in different regions. The results show that directional antennas deployed at both TX and RX can significantly improve the coverage range of mobile communication system. But the directional antennas will bring about larger decorrelation distance in radio channel. Therefore, the interval between antennas at RX (if multiantennas are applied) should be separated by more than $2.5 \mathrm{~m}$ to overcome deep shadow fading. Meanwhile, the directional antennas play the role of spatial filter which obviously attenuates the rays out of the antenna main lobe, especially when RX is close to TX. These effects greatly decrease RMS delay spread in the near region. Actually, comparing to omnidirectional antenna, the directional antenna mostly affects the channel characteristics in the near region, but it rarely affects the channel characteristics in the far region. Considering the length of the near region is around $50 \mathrm{~m}$ in the study, the HSR will get through the near region in a flash ( 0.5 second). In this moment when the channel changes with extreme rapidity, it is difficult to keep a reliable communication link. So, advanced handover strategies should be considered before or after HSR getting through the near region. Finally, the vertical polarized antenna is suggested in this arched tunnel rather than horizontal polarized antenna. 


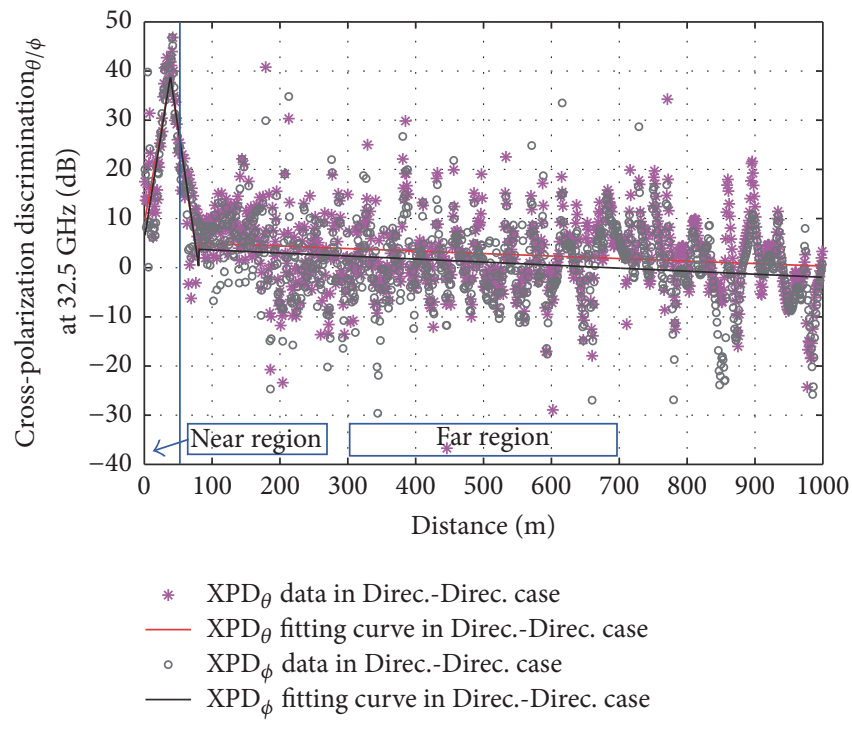

(a)

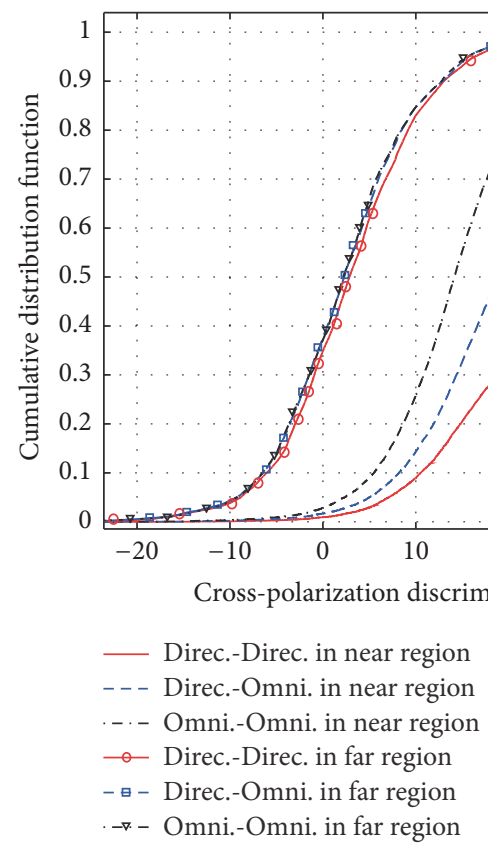

(b)

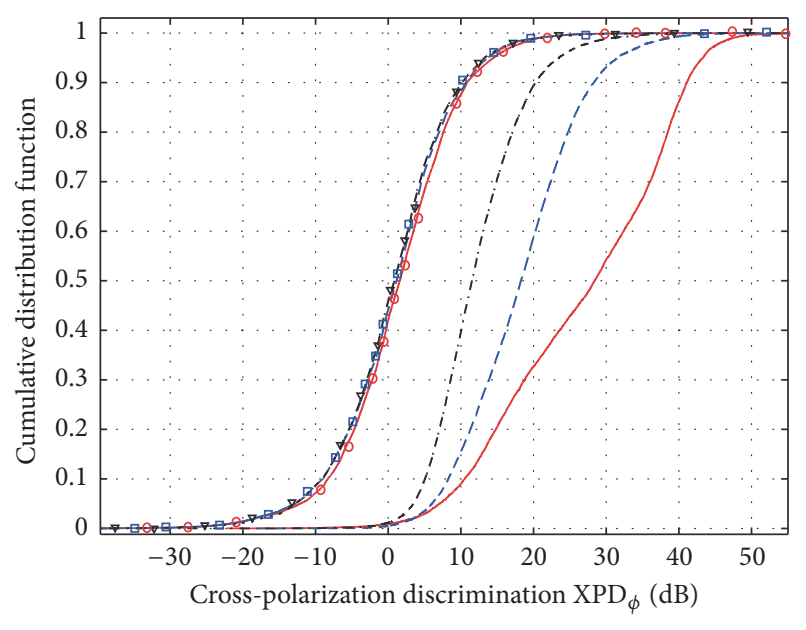

— Direc.-Direc. in near region

- - Direc.-Omni. in near region

. - Omni.-Omni. in near region

$\rightarrow$ Direc.-Direc. in far region

- - Direc.-Omni. in far region

- - - Omni.-Omni. in far region

(c)

Figure 19: (a) Results of $\mathrm{XPD}_{\theta}$ and $\mathrm{XPD}_{\varphi}$ in the Direc.-Direc. case at $32.5 \mathrm{GHz}$. Two fitted lines describe the variation tendencies for XPD ${ }_{\theta}$ and $X P D_{\varphi}$, respectively. (b) CDFs of $\mathrm{XPD}_{\theta}$ in different antenna setups and regions at whole frequencies. (c) $\mathrm{CDFs}_{\mathrm{O}}$ of $\mathrm{XPD}_{\varphi}$ in different antenna setups and regions at whole frequencies.

\section{Conflicts of Interest}

The authors declare that there are no conflicts of interest regarding the publication of this paper.

\section{Acknowledgments}

This work is supported by Institute for Information \& Communications Technology Promotion (IITP) grant funded by the Korea government (MSIT) (no. 2014-0-00282, Development of $5 \mathrm{G}$ Mobile Communication Technologies for HyperConnected Smart Services).

\section{References}

[1] Z. Pi and F. Khan, "An introduction to millimeter-wave mobile broadband systems," IEEE Communications Magazine, vol. 49, no. 6, pp. 101-107, 2011.

[2] B. Ai, K. Guan, M. Rupp et al., "Future railway services-oriented mobile communications network," IEEE Communications Magazine, vol. 53, no. 10, pp. 78-85, 2015.

[3] K. Guan, G. Li, T. Kuerner et al., "On millimeter wave and THz mobile radio channel for smart rail mobility," IEEE Transactions on Vehicular Technology, vol. 66, no. 7, pp. 5658-5674, 2016.

[4] B. Ai, X. Cheng, T. Kurner et al., "Challenges toward wireless communications for high-speed railway," IEEE Transactions on 
Intelligent Transportation Systems, vol. 15, no. 5, pp. 2143-2158, 2014.

[5] Q. Wang, B. Ai, D. W. Matolak et al., "Spatial variation analysis for measured indoor massive MIMO channels," IEEE Access, 2017.

[6] T. Rappaport, S. Sun, R. Mayzus et al., "Millimeter wave mobile communications for $5 \mathrm{G}$ cellular: it will work!", IEEE Access, vol. 1, pp. 335-349, 2013.

[7] T. S. Rappaport, Y. Xing, G. R. MacCartney, A. F. Molisch, E. Mellios, and J. Zhang, "Overview of millimeter wave communications for fifth-generation (5G) wireless networks-with a focus on propagation models," IEEE Transactions on Antennas and Propagation, 2017.

[8] J. Zhang, P. Tang, L. Tian, Z. Hu, T. Wang, and H. Wang, "6-100 GHz research progress and challenges from a channel perspective for fifth generation (5G) and future wireless communication," Science China Information Sciences, vol. 60, no. 8, Article ID 080301, 2017.

[9] G. Li, K. Guan, B. Ai et al., "On the high-speed railway communication at $30 \mathrm{GHz}$ band: Feasibility and channel characteristics," in Proceedings of the 11th International Symposium on Antennas, Propagation and EM Theory, ISAPE 2016, pp. 796799, October 2016.

[10] J. Wang, H. Zhu, and N. J. Gomes, "Distributed antenna systems for mobile communications in high speed trains," IEEE Journal on Selected Areas in Communications, vol. 30, no. 4, pp. 675-683, 2012.

[11] X. Chen, S. Guo, and Q. Wu, "Link-level analysis of a multiservice indoor distributed antenna system [wireless corner]," IEEE Antennas and Propagation Magazine, vol. 59, no. 3, pp. 154-162, 2017.

[12] W. Fan, T. Jamsa, J. O. Nielsen, and G. F. Pedersen, "On angular sampling methods for 3-D spatial channel models," IEEE Antennas and Wireless Propagation Letters, vol. 14, pp. 531-534, 2015.

[13] M. V. S. N. Prasad, R. Singh, S. K. Sarkar, and A. D. Sarma, "Some experimental and modeling results of widely varying urban environments on train mobile radio communication," Wireless Communications and Mobile Computing, vol. 6, no. 1, pp. 105-112, 2006.

[14] K. Guan, B. Ai, Z. Zhong et al., "Measurements and Analysis of Large-Scale Fading Characteristics in Curved Subway Tunnels at $920 \mathrm{MHz}, 2400 \mathrm{MHz}$, and $5705 \mathrm{MHz}$," IEEE Transactions on Intelligent Transportation Systems, vol. 16, no. 5, pp. 2393-2405, 2015.

[15] J. Kim, H.-S. Chung, I. G. Kim, H. Lee, and M. S. Lee, "A study on millimeter-wave beamforming for high-speed train communication," in Proceedings of the 6th International Conference on Information and Communication Technology Convergence, ICTC 2015, pp. 1190-1193, October 2015.

[16] K. Guan, Z. Zhong, B. Ai, and T. Kürner, "Deterministic propagation modeling for the realistic high-speed railway environment," in Proceedings of the IEEE 77th Vehicular Technology Conference, VTC Spring 2013, June 2013.

[17] T. Abbas, J. Nuckelt, T. Zemen, C. F. Mecklenbräuker, and F. Tufvesson, "Simulation and measurement-based vehicle-tovehicle channel characterization: accuracy and constraint analysis," IEEE Transactions on Antennas and Propagation, vol. 63, no. 7, pp. 3208-3218, 2015.

[18] Q. Wang, B. Ai, K. Guan, Y. Li, and Z. Zhong, "Ray-based analysis of small-scale fading for indoor corridor scenarios at
$15 \mathrm{GHz}$," in Proceedings of the Asia-Pacific Symposium on Electromagnetic Compatibility (APEMC '15), pp. 181-184, IEEE, Taipei, Taiwan, May 2015.

[19] S. Priebe and T. Kurner, "Stochastic modeling of THz indoor radio channels," IEEE Transactions on Wireless Communications, vol. 12, no. 9, pp. 4445-4455, 2013.

[20] J. W. McKown and R. L. Hamilton Jr., "Ray tracing as a design tool for radio networks," IEEE Network, vol. 5, no. 6, pp. 27-30, 1991.

[21] J. Nuckelt, M. Schack, and T. Kürner, "Deterministic and stochastic channel models implemented in a physical layer simulator for Car-to-X communications," Advances in Radio Science, vol. 9, pp. 165-171, 2011.

[22] V. Degli-Esposti, F. Fuschini, E. M. Vitucci, and G. Falciasecca, "Measurement and modelling of scattering from buildings," IEEE Transactions on Antennas and Propagation, vol. 55, no. 1, pp. 143-153, 2007.

[23] W. Fan, I. Carton, P. Kyösti, and G. . Pedersen, "Emulating ray-tracing channels in multiprobe anechoic chamber setups for virtual drive testing," IEEE Transactions on Antennas and Propagation, vol. 64, no. 2, pp. 730-739, 2016.

[24] R. C. Jones, "A new calculus for the treatment of optical systems: I. description and discussion of the calculus," Journal of the Optical Society of America, vol. 31, no. 7, pp. 488-493, 1941.

[25] "Recommendation itu-r p.1238-8-em property," https://www .itu.int/rec/R-REC-P.1238/en.

[26] J. Pascual-Garcia, J. Molina-Garcia-Pardo, M. Martinez-Ingles, J. Rodriguez, and N. Saurin-Serrano, "On the importance of diffuse scattering model parameterization in indoor wireless channels at mm-wave frequencies," IEEE Access, vol. 4, pp. 688701, 2016.

[27] S. Priebe, M. Kannicht, M. Jacob, and T. Kurner, "Ultra broadband indoor channel measurements and calibrated ray tracing propagation modeling at $\mathrm{THz}$ frequencies," Journal of Communications and Networks, vol. 15, no. 6, pp. 547-558, 2013.

[28] D. K. Ghodgaonkar, V. V. Varadan, and V. K. Varadan, "A free-space method for measurement of dielectric constants and loss tangents at microwave frequencies," IEEE Transactions on Instrumentation and Measurement, vol. 38, no. 3, pp. 789-793, 1989.

[29] A. Corana, M. Marchesi, C. Martini, and S. Ridella, "Minimizing multimodal functions of continuous variables with the "simulated annealing" algorithm," Association for Computing Machinery. Transactions on Mathematical Software, vol. 13, no. 3, pp. 262-280, 1987.

[30] K. Guan, Z. D. Zhong, J. I. Alonso, and C. Briso-Rodríguez, "Measurement of distributed antenna systems at $2.4 \mathrm{GHz}$ in a realistic subway tunnel environment," IEEE Transactions on Vehicular Technology, vol. 61, no. 2, pp. 834-837, 2012.

[31] A. F. Molisch, Wireless Communications, vol. 34, John Wiley \& Sons, 2012.

[32] J. Nuckelt, M. Schack, and T. Kürner, "Geometry-based path interpolation for rapid ray-optical modeling of vehicular channels," in Proceedings of the 9th European Conference on Antennas and Propagation, EuCAP 2015, pp. 1-5, May 2015.

[33] G. Li, B. Ai, K. Guan et al., "Channel characterization for mobile hotspot network in subway tunnels at $30 \mathrm{GHz}$ band," in Proceedings of the 83rd IEEE Vehicular Technology Conference, VTC Spring 2016, May 2016.

[34] G. Li, B. Ai, K. Guan et al., "Path loss modeling and fading analysis for channels with various antenna setups in tunnels at 
$30 \mathrm{GHz}$ band," in Proceedings of the 10th European Conference on Antennas and Propagation (EuCAP '16), April 2016.

[35] W. C. Y. Lee, "Estimate of local average power of a mobile radio signal," IEEE Transactions on Vehicular Technology, vol. 34, no. 1, pp. 22-27, 1985.

[36] R. He, B. Ai, Z. Zhong, A. F. Molisch, R. Chen, and Y. Yang, "A measurement-based stochastic model for high-speed railway channels," IEEE Transactions on Intelligent Transportation Systems, vol. 16, no. 3, pp. 1120-1135, 2015.

[37] A. F. Molisch, Wireless Communications, John Wiley \& Sons, 2007.

[38] T. S. Rappaport, Wireless Communications: Principles and Practice, vol. 2, Prentice Hall PTR, Upper Saddle River, NJ, 1996.

[39] S. Priebe, "Towards THz Communications: Propagation Studies, Indoor Channel Modeling and Interference Investigations," 2013.

[40] A. Panahandeh, F. Quitin, J. M. Dricot, F. Horlin, C. Oestges, and P. De Doncker, "Multi-Polarized Channel Statistics for Outdoor-to-Indoor and Indoor-to-Indoor Channels," in Proceedings of the IEEE 71st Vehicular Technology Conference, pp. 1-5, Taipei, Taiwan, May 2010. 


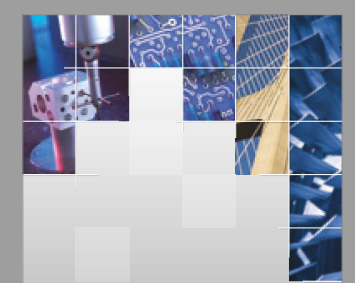

\section{Enfincering}
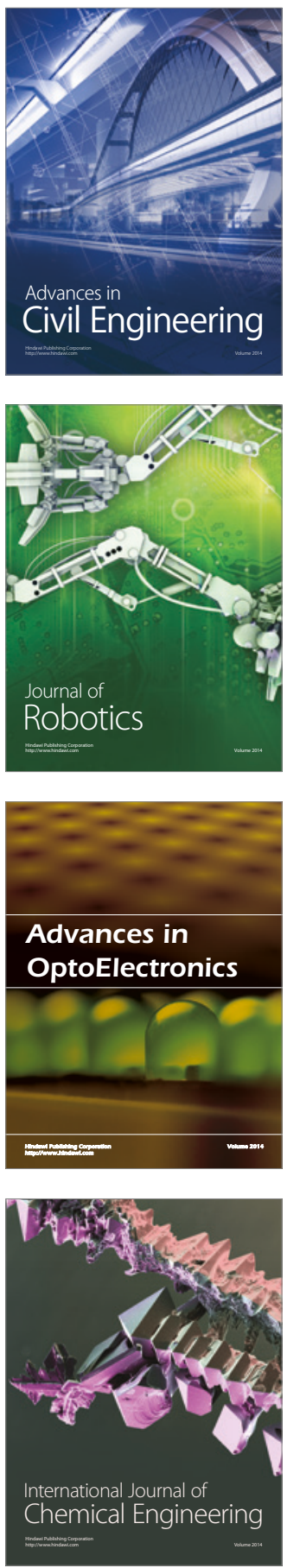

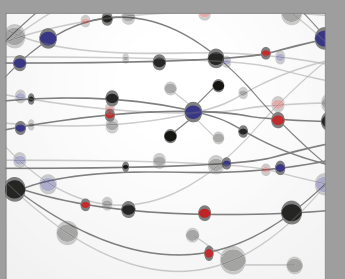

The Scientific World Journal

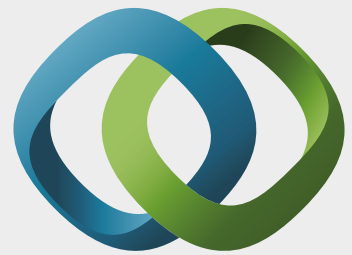

\section{Hindawi}

Submit your manuscripts at

https://www.hindawi.com
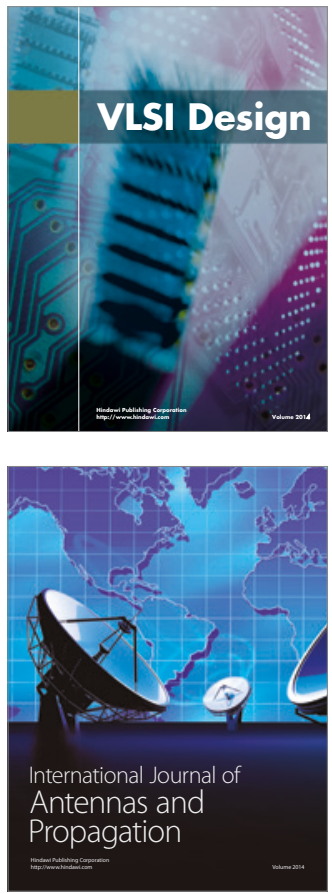

\section{Rotating}

Machinery
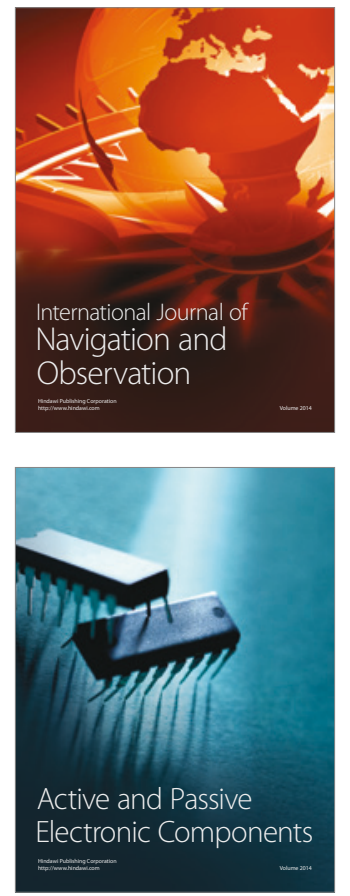
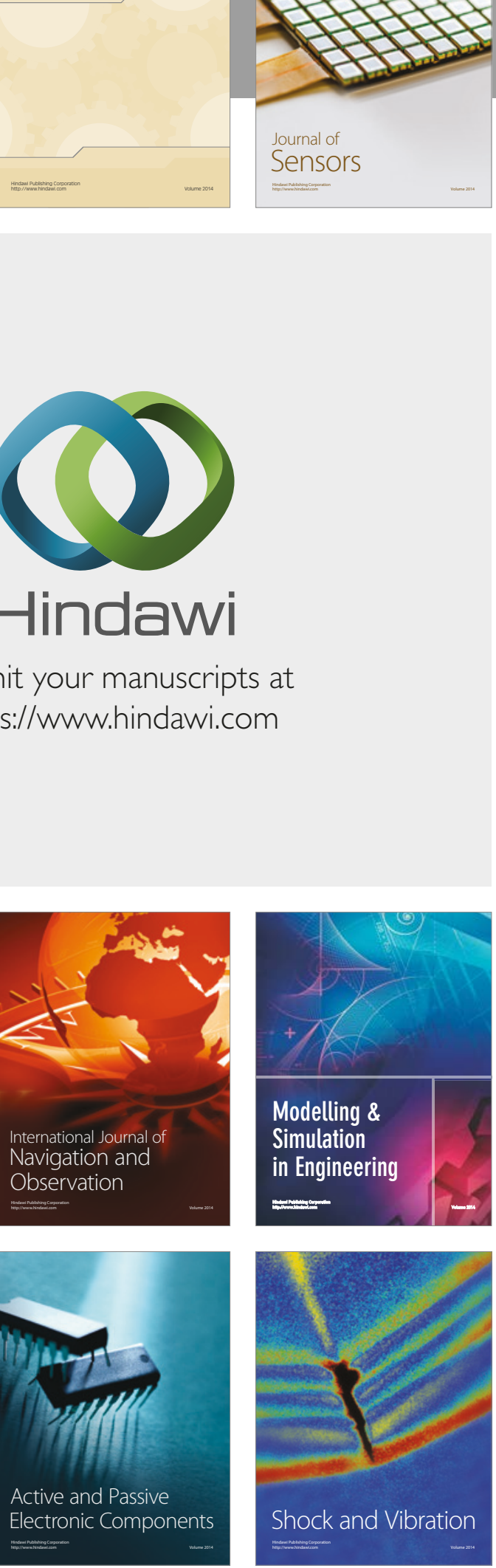
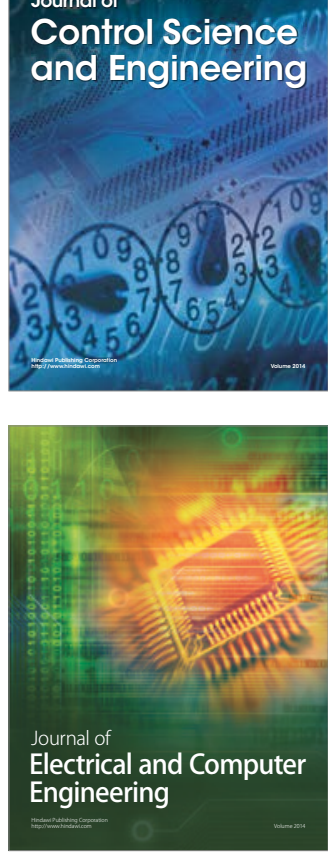

Distributed

Journal of

Control Science

and Engineering
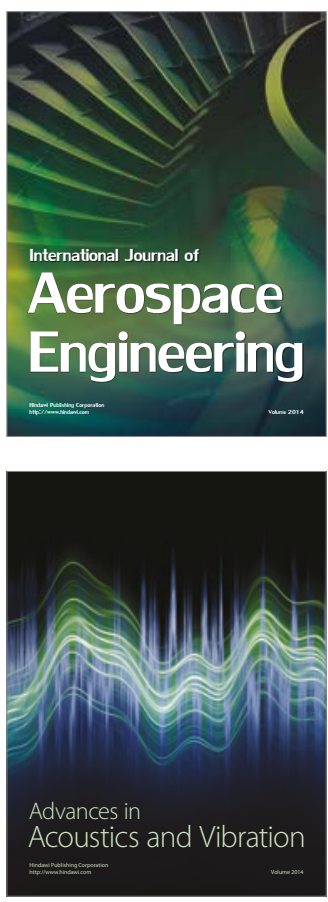

Sensor Networks 